\title{
ヘь
}

Descriptive Set Theory and Harmonic Analysis

Author(s): A. S. Kechris and A. Louveau

Source: The Journal of Symbolic Logic, Vol. 57, No. 2 (Jun., 1992), pp. 413-441

Published by: Association for Symbolic Logic

Stable URL: http://www.jstor.org/stable/2275277

Accessed: 16/05/2013 17:56

Your use of the JSTOR archive indicates your acceptance of the Terms \& Conditions of Use, available at http://www.jstor.org/page/info/about/policies/terms.jsp

JSTOR is a not-for-profit service that helps scholars, researchers, and students discover, use, and build upon a wide range of content in a trusted digital archive. We use information technology and tools to increase productivity and facilitate new forms of scholarship. For more information about JSTOR, please contact support@jstor.org. 


\section{DESCRIPTIVE SET THEORY AND HARMONIC ANALYSIS}

\section{A. S. KECHRIS AND A. LOUVEAU}

Introduction. During the 1989 European ASL Summer Meeting in Berlin, the authors gave a series of eight lectures (short course) on the topic of the title. This survey article consists basically of the lecture notes for that course distributed to the participants of that conference. We have purposely tried in this printed version to preserve the informal style of the original notes.

Let us say first a few things aboui the content of these lectures. Our aim has been to present some recent work in descriptive set theory and its applications to an area of harmonic analysis. Typical uses of descriptive set theory in analysis are most often through regularity properties of definable sets, like measurability, the property of Baire, capacitability, etc., which are used to show that certain problems have solutions that behave nicely. In the theory we will present, definability itself, in fact the precise analysis of the "definable complexity" of certain sets, will be the main concern. It will be through such knowledge that we will be able to infer important structural properties of various objects which will then be used to solve analysis problems.

The first lecture provides a short historical introduction to the subject of uniqueness for trigonometric series, which is the area of harmonic analysis whose problems are the origin of this work. As is well known, it was Cantor who proved the first major result in this subject in 1870, and it was his subsequent work here that led him to the creation of set theory.

The next three lectures describe the recently developed definability theory of $\sigma$-ideals of closed sets, which is the main tool through which descriptive set theory is applied to the analysis problems we are interested in. Proofs or sketches of proofs are given here for most of the main facts, especially those that are used later on.

In the last four lectures, we first present an outline of the analytical theory of uniqueness of trigonometric series that is needed here. In order to give a bit of the flavor of the subject, we also give here some sketches of the less technical or more

Received May 29, 1991.

The research of the first author was partially supported by NSF grant DMS-9020153. 
elementary arguments (including for example Cantor's proof). Finally we show how the analysis can be combined with the descriptive set theory to produce several applications to the analysis problems. We conclude with a summary of the most recent work in this area.

Lecture I. A bit of history. Classical harmonic analysis is the study of periodic phenomena, which can be represented by mathematical objects, like functions, measures, distributions etc., on the circle $\mathbb{T}$ (identified with $\mathbb{R} / 2 \pi \mathbb{Z}$ ), and their analysis in terms of "harmonics", i.e. trigonometric series of the form $\sum_{n \in \mathbf{Z}} c_{n} e^{i n x}$, where the coefficients $c_{n}$ are complex numbers and $x$ varies over $\mathbb{R}$.

This study naturally divides into convergence questions (of the series to the represented object), uniqueness questions of the series representing an object, and computation of the series, if uniqueness holds.

Let us give some examples:

1 (Uniform convergence). If $\sum_{n}\left|c_{n}\right|<\infty$, the partial sums $\sum_{-N}^{N} c_{n} e^{i n x}$ uniformly converge on $\mathbb{T}$ to a continuous function (the set of such functions is denoted $A(\mathbb{T})$ ). Moreover, for $f$ in $A(\mathbb{T})$ its corresponding series is unique, and the $n$th coefficient is given by the Fourier transform

$$
c_{n}=\hat{f}(n)=\int_{\mathbf{T}} f(x) e^{-i n x} d \lambda,
$$

where $\lambda$ denotes the normalized Lebesgue measure on $\mathbb{T}$.

2 (Convergence in $L^{2}$ ). If $f \in L^{2}(\mathbb{T})$, the series $\sum_{-\infty}^{\infty} c_{n} e^{i n x}$ converges in $L^{2}$ to $f$, with uniqueness.

The notion of convergence of trigonometric series we will be interested in is pointwise convergence everywhere. Note that the limit is then of the first Baire class, but may not be integrable, so that the Fourier transform makes no sense.

Around the middle of the 19th century, Riemann and Heine posed the uniqueness problem for this notion of convergence: Suppose $\sum c_{n} e^{i n x}$ and $\sum d_{n} e^{i n x}$ are two series which converge for all $x \in \mathbb{R}$ to the same function $f(x)$. Does one have necessarily $c_{n} \equiv d_{n}$ ?

Equivalently, if $\sum_{n} c_{n} e^{i n x}=0$ everywhere on $\mathbb{R}$, is $c_{n}$ identically zero?

Cantor (1870): Yes.

Cantor (1872), essentially: Yes, even if one relaxes the hypothesis to: $\sum c_{n} e^{i n x}$ converges to 0 , except maybe on a closed countable set of $x$ 's.

This last result leads naturally to the following

Definition. A set $E \subseteq \mathbb{T}$ is a set of uniqueness if every trigonometric series $\sum c_{n} e^{i n x}$ which converges to 0 outside the set $E$ is in fact identically 0 . Otherwise it is a set of multiplicity.

$\mathscr{U}$ will denote the family of sets of uniqueness, $U=\mathscr{U} \cap K(\mathbb{T})$ the family of closed uniqueness sets. (Here $K(\mathbb{T})=$ the class of closed subsets of $\mathbb{T}$.)

So Cantor's result can be rephrased as $K_{\omega}(\mathbb{T}) \subseteq U$, where $K_{\omega}(\mathbb{T})$ denotes the countable closed subsets of $\mathbb{T}$.

The $U$-sets are small, exceptional sets. For example, the $U$-sets (in fact the Borel $\mathscr{U}$-sets) are of Lebesgue measure 0 . 
This gives

$$
K_{\omega}(\mathbb{T}) \subseteq U \subseteq \text { Lebesgue measure } 0
$$

These inclusions are proper:

Bary and Rajchman (1921-1923). There are perfect $U$-sets. In fact, the classical Cantor $\frac{1}{3}$-set is a set of uniqueness.

Menshov (1916). There are closed multiplicity sets of Lebesgue measure 0 (and hence nonzero trigonometric series which converge to 0 a.e.).

In his proof, Menshov builds a probability measure $\mu$, with closed support $E$ of Lebesgue measure 0 , such that the Fourier coefficients $\hat{\mu}(n)=\int e^{-i n x} d \mu(x) \rightarrow 0$ as $|n| \rightarrow \infty$. This easily implies that $\sum \hat{\mu}(n) e^{i n x}$ converges to 0 off $E$. So multiplicity is witnessed by the Fourier transform of a measure. This leads to the next definition.

DEFINITION. A set $E$ is of restricted multiplicity if multiplicity is witnessed by (the Fourier transform of) a measure, i.e. if there is a measure $\mu \neq 0$ such that $\sum \hat{\mu}(n) e^{i n x}$ converges to 0 outside $E$. Otherwise, it is called a set of extended uniqueness. The family of sets of extended uniqueness is denoted $\mathscr{U}_{0}$, and $U_{0}=\mathscr{U}_{0} \cap K(\mathbb{T})$ is the family of closed sets of extended uniqueness.

The picture is then

$$
K_{\omega}(\mathbb{T}) \varsubsetneqq U \varsubsetneqq U_{0} \varsubsetneqq \text { Lebesgue measure 0, }
$$

the inequality $U \neq U_{0}$ being a much later result of Piatetski-Shapiro (1953).

By their very definitions, $\mathscr{U}$ and $\mathscr{U}_{0}$ are hereditary, i.e. if $F \subseteq$ a $\mathscr{U}$-set $E$, then $F$ is a $\mathscr{U}$-set, and similarly for $\mathscr{U}_{0}$. An important closure property is the following:

Bary (1923). If $\left(E_{n}\right)_{n \in \omega}$ are closed sets in $U$, then $E=\bigcup_{n} E_{n}$ is a $\mathscr{U}$-set (and hence if $E$ is closed, $E \in U$-i.e. $U$ is what is called a $\sigma$-ideal of closed sets).

By the 1920's it had become clear that the concept of a set of uniqueness was quite difficult to delineate. In her 1927 memoir on this subject, Bary [1] included some basic problems on $\mathscr{U}$ :

1. The Union Problem. Is the union of two (or countably many) Borel $\mathscr{U}$-sets a $\mathscr{U}$-set? (Easily true for $\mathscr{U}_{0}$; open even for two $G_{\delta}$ 's.)

2. The Interior Problem. If all closed subsets of a Borel set $B$ are of uniqueness, is $B$ also of uniqueness? (Easily true for $\mathscr{U}_{0}$; open even for $G_{\delta}$ 's.)

3. The Category Problem. Every Borel $\mathscr{U}$-set (or even $\mathscr{U}_{0}$-set) is of Lebesgue measure 0 . Is every Borel $\mathscr{U}$-set of the first category?

4. The Characterization Problem. Find some "structural" criteria for deciding whether a given perfect set $E$ is in $U$ or not. This is a rather vague heuristic problem. Somehow its intended meaning seems to have been that of asking for geometric, analytic, or, as we will see later on, even number-theoretic properties of a perfect set $E$, expressed explicitly in terms of some standard specification of $E$, like for example its contiguous intervals, that will determine whether it is a uniqueness or multiplicity set.

We will see below how recent work has thrown some light on these and other problems in this area. But first we will discuss the descriptive theory of $\sigma$-ideals of closed sets. 
Lecture II. Complexity of $\sigma$-ideals of closed sets. For any topological space $A$, $K(A)$ denotes the set of compact subsets of $A$.

For the sequel, $E$ denotes a compact metric space, with distance $d$. The space $K(E)$ is then metrized as follows: Given $K \in K(E)$ and $\varepsilon>0$, the $\varepsilon$-neighborhood of $K$, denoted by $B(K, \varepsilon)$, is $\bigcup\{B(x, \varepsilon): x \in K\}=\{y: \exists x \in K(d(x, y)<\varepsilon)\}$. One defines the Hausdorff distance $\delta$ on $K(E)$ by

$$
\delta(K, L)=\inf \{\varepsilon>0: K \in B(L, \varepsilon) \text { and } L \in B(K, \varepsilon)\}
$$

if $K, L \neq \varnothing$, while $\delta(K, \varnothing)=\sup (d)$ if $K \neq \varnothing, 0$ if $K=\varnothing$. We have

(i) If $S \subseteq E$ is finite with $B(S, \varepsilon / 2)=E$, then $K(S)$ is a finite set in $K(E)$ with $B(K(S), \varepsilon)=K(E)$, so that $K(E)$ is totally bounded.

(ii) If $\left(K_{n}\right)$ is a Cauchy sequence with $\delta\left(K_{n}, K_{n+1}\right) \leq 2^{-n-1}$, then $K=\bigcap_{n} \overline{B\left(K_{n}, 2^{n}\right)}$ is the limit of the sequence $\left(K_{n}\right)$, so that $K(E)$ is complete. This proves

FACT $1 . K(E)$ is a compact metric space for the Hausdorff metric $\delta$.

The topology can also be described easily:

FACT 2. (i) If $\left(V_{n}\right)$ is a basis of open sets for E closed under finite unions, the sets

$$
\begin{gathered}
W_{n_{0}, n_{1}, \ldots, n_{p}}=\left\{K: K \subseteq V_{n_{0}} \text { and } K \cap V_{n_{1}} \neq \varnothing \text { and } K \cap V_{n_{2}} \neq \varnothing\right. \\
\text { and } \left.\ldots \text { and } K \cap V_{n_{p}} \neq \varnothing\right\}
\end{gathered}
$$

form a basis for $K(E)$.

(ii) If $D$ is dense in $E, K_{<\omega}(D)=\{$ finite subsets of $D\}$ is dense in $K(E)$.

FACT 3. (i) For $L \in K(E), K(L)$ is closed and $K(E \backslash L)$ is open.

(ii) $\cup: K(E) \times K(E) \rightarrow K(E)$ is continuous, but $\cap$ is generally not. More generally, if $K(K(E))$ is given the Hausdorff topology too, then $\bigcup: K(K(E)) \rightarrow K(E)$ is continuous (where for $L \in K(K(E)$ ), we put $\bigcup L=\bigcup\{K: K \in L\}$, which is easily in $K(E)$.)

(iii) $\{\cdot\}: E \rightarrow K(E)$ is an isometry, and $\in$ and $\subseteq$ are closed relations.

(iv) If $f: E \rightarrow F$ is continuous, so is $f^{\prime \prime}: K(E) \rightarrow K(F)$.

Note that if $E$ is perfect, $K(E) \backslash\{\varnothing\}$ is perfect too, but $\varnothing$ is always isolated in $K(E)$. Also, if $E$ is dim 0 (i.e. has a basis of clopen sets), so is $K(E)$. In fact for $E=2^{\omega}, K(E)$ is homeomorphic to a compact subspace of $2^{\text {Seq } \omega}$ (where Seq $\omega=\omega^{<\omega}$ ), via the usual map which to $K \in K(E)$ associates its canonical tree $T(K)=\{s \in \operatorname{Seq} \omega$ : $\exists \alpha \in K(s \subseteq \alpha)\}$.

DEFINITION. Let $I$ be a subset of $K(E)$.

$I$ is hereditary (among closed sets) if $L \in I$ and $K \in K(E), K \subseteq L \Rightarrow K \in I$.

$I$ is an ideal (of closed sets) if $I$ is hereditary and closed under finite unions.

$I$ is a $\sigma$-ideal (of closed sets) if $I$ is hereditary and closed under countable unions which are closed, i.e. if for any sequence $\left(K_{n}\right)$ in $I$ and any $K \in K(E), K \subseteq \bigcup_{n} K_{n} \Rightarrow$ $K \in I$.

$I$ is an $\infty$-ideal if $I$ is hereditary and closed under arbitrary unions which are closed.

We will use a similar terminology for other families, like $G_{\delta}$, Borel, etc.

$\infty$-ideals are easy to describe: If we let $S_{I}$, the support of $I$, be $\{x \in E:\{x\} \in I\}$, then $I$ is an $\infty$-ideal $\Leftrightarrow I=K\left(S_{I}\right)$.

There are many different kinds of $\sigma$-ideals. Here are some examples:

1. For $A \subseteq E, K(A)$ and $K_{\omega}(A)=\{K \in K(A): K$ is countable $\}$. 
2. $I_{m}=\{K: K$ is meager (= nowhere dense) $\}$.

3. In measure theory: For $\mu$ a measure on $E, I_{\mu}=\{K: \mu(K)=0\}$ and, more generally, for a Choquet subadditive capacity $\gamma$, a Hausdorff measure $\mu^{h}$ or a set $H$ of measures, $I_{\gamma}=\{K: \gamma(K)=0\}, I_{\mu^{h}}=\left\{K: \mu^{h}(K)=0\right\}, I_{H}=\{K: \forall \mu \in H(\mu(K)=0)\}$.

4. $J_{\gamma}=\{K: K$ is $\gamma$-thin $\}$, where

$$
\begin{aligned}
K \text { is } \gamma \text {-thin } \Leftrightarrow & \text { Every family of pairwise disjoint compact subsets } \\
& \text { of } K \text { not in } I_{\gamma} \text { is countable }
\end{aligned}
$$

and, with similar definitions,

$$
J_{\mu^{h}}=\left\{K: K \text { is } \mu^{h} \text {-thin }\right\}, \quad J_{H}=\{K: K \text { is } H \text {-thin }\} .
$$

5. In harmonic analysis, $U$ and $U_{0}$.

6. $I_{c}=\left\{K \subseteq 2^{\omega}: K\right.$ avoids a cone of Turing degrees $\}$.

7. For $B$ a separable Banach space, and $E=B_{1}^{*}$ the unit ball of its dual with the weak ${ }^{*}$-topology, $I_{\text {sep }}=\left\{K \subseteq B_{1}^{*}: K\right.$ is norm separable $\}$.

We want to study the possible complexities of $\sigma$-ideals of closed sets. These will be measured by their descriptive Wadge class.

Recall that a class (or pointclass) is a family of sets (in Polish spaces) closed under preimages by continuous functions. If $A$ is a set, its Wadge class $\Gamma(A)$ is the class generated by $A$, i.e. the family of continuous preimages of $A$. Given a class $\Gamma$, we say that $A$ is $\Gamma$-hard if every subset $B$ of $2^{\omega}$ in $\Gamma$ is a continuous preimage of $A$, and that $A$ is $\Gamma$-complete if $A$ is $\Gamma$-hard and $A \in \Gamma$. Finally $A$ is a true $\Gamma$-set if $A \in \Gamma$ but $A \notin \check{\Gamma}$, the dual class of $\Gamma$, consisting of all complements of sets in $\Gamma$.

We want to compute all $\Gamma(I), I$ a $\sigma$-ideal of closed sets in some $K(E), E$ compact metric. Within the class $\boldsymbol{\Sigma}_{1}^{1} \cup \boldsymbol{\Pi}_{1}^{1}$, this is given by

THEOREM 1 (Kechris, Louveau and Woodin [23]). Let $I \subseteq K(E)$ be a $\sigma$-ideal in $\Sigma_{1}^{1} \cup \boldsymbol{\Pi}_{1}^{1}$. Then one of the following holds:

(i) $I$ is $\boldsymbol{\Pi}_{1}^{1}$-complete.

(ii) $I$ is $\Pi_{2}^{0}$-complete.

(iii) I is $\boldsymbol{D}_{2}$-complete, where $\boldsymbol{D}_{2}$ is the class of differences of two closed sets.

(iv) $I$ is $\Pi_{1}^{0}$-complete.

(v) I is $\Sigma_{1}^{0}$-complete.

(vi) $I$ is $\Delta_{1}^{0}$, i.e. clopen.

Moreover, if $I$ is not an $\infty$-ideal, i.e. is not of form $K(A)$, then only (i) or (ii) can occur (the dichotomy theorem).

The main consequence of this result is that $\Pi_{1}^{1} \sigma$-ideals fall into one of two categories: the simple ones $\left(\boldsymbol{\Pi}_{2}^{0}\right)$, and the complicated ones $\left(\boldsymbol{\Pi}_{1}^{1}\right.$-complete).

Examples of "simple" $\left(\Pi_{2}^{0}\right) \sigma$-ideals include $K(A)$ for $A \in \Pi_{2}^{0} ; I_{m} ; I_{\mu} ; I_{\gamma} ; I_{\mu h} ; I_{H}$ for $H$ in $\Sigma_{2}^{0}$; some $J_{\gamma}$ 's and $J_{H}$ 's.

Examples of "complicated" ( $\boldsymbol{\Pi}_{1}^{1}$-complete) $\sigma$-ideals include $K(A)$ for $A \in \boldsymbol{\Pi}_{1}^{1}$, $A \notin \Pi_{2}^{0} ; K_{\omega}\left(2^{\omega}\right)$; some $I_{H}$ 's, $J_{\gamma}$ 's and $J_{H}$ 's; $U$ and $U_{0} ; I_{\text {sep }} ; I_{c}$.

The proof of the theorem needs four steps:

(1) To exhibit $\sigma$-ideals (in fact $\infty$-ideals) complete in each class.

(2) To prove "Hurewicz-type" results which will reduce any $\boldsymbol{\Pi}_{1}^{1} \sigma$-ideal to one of the six examples. 
(3) To prove that any $\boldsymbol{\Delta}_{2}^{0} \sigma$-ideal is an $\infty$-ideal.

(4) To prove that any $\Sigma_{1}^{1} \sigma$-ideal is $\Pi_{2}^{0}$.

We will concentrate on (1) and (2). Let us just say that (3) can be improved to show that any $\boldsymbol{\Delta}_{2}^{0}$ ideal is an $\infty$-ideal, and also any $\boldsymbol{\Pi}_{2}^{0}$-ideal is a $\sigma$-ideal. And (4) uses ideas of Christensen and Saint-Raymond, who proved that every $\Sigma_{1}^{1} \infty$-ideal is $\Pi_{2}^{0}$.

Step 1. Let $A$ be a subset of $E \times 2^{\omega}$, and define

$$
\forall A=\left\{x \in E: \forall \alpha \in 2^{\omega}[(x, \alpha) \in A]\right\} .
$$

If $\Gamma$ is a class, define

$$
\forall \Gamma=\{\forall A: A \in \Gamma\} .
$$

LEMma 2. Let $\Gamma$ be a class, and $A \subseteq E$ be $\Gamma$-hard. Then $K(A)$ is $\forall \Gamma$-hard in $K(E)$.

Proof. Let $B \subseteq 2^{\omega}$ be any set in $\forall \Gamma$, and let $C \subseteq 2^{\omega} \times 2^{\omega}$ be in $\Gamma$, with $B=\forall C$. As $A$ is $\Gamma$-hard, there exists a continuous $f: 2^{\omega} \times 2^{\omega} \rightarrow E$ with $C=f^{-1}(A)$. Define $\varphi: 2^{\omega} \rightarrow K(E)$ by $\varphi(x)=\left\{f(x, \alpha): \alpha \in 2^{\omega}\right\}$. Then $\varphi$ is continuous, and one checks that $\varphi^{-1}(K(A))=B$.

LEMMA 3.

$$
\begin{array}{ll}
\boldsymbol{\Pi}_{1}^{1}=\forall \Sigma_{2}^{0}, & \boldsymbol{\Pi}_{2}^{0}=\forall \check{\boldsymbol{D}}_{2}, \\
\boldsymbol{D}_{2}=\forall \Delta\left(\boldsymbol{D}_{2}\right), & \boldsymbol{\Pi}_{1}^{0}=\forall \boldsymbol{\Pi}_{1}^{0}, \\
\boldsymbol{\Sigma}_{1}^{0}=\forall \Sigma_{1}^{0}, & \boldsymbol{\Delta}_{1}^{0}=\forall \Delta_{1}^{0}
\end{array}
$$

(where $\Delta(\Gamma)=\Gamma \cap \check{\Gamma})$.

Proof. Easy.

This lemma says that the six classes of Theorem 1 are exactly, within $\Sigma_{1}^{1} \cup \Pi_{1}^{1}$, the classes of form $\forall \Gamma$.

LemMA 4. (i) (Hurewicz [6]). Let $D$ be a countable dense subset of $2^{\omega}$. Then $D$ is $\Sigma_{2}^{0}$-complete, and $K(D)$ is $\boldsymbol{\Pi}_{1}^{1}$-complete.

(ii) Let $A_{2}=\{\omega \cdot n+p: n \in \omega, p>0\} \cup\left\{\omega^{2}\right\}$ in $E_{2}=\omega^{2}+1$. Then $A_{2}$ is $\check{D}_{2}$ complete, and hence $K\left(A_{2}\right)$ is $\Pi_{2}^{0}$-complete.

(iii) Let $A_{3}=\omega \cup\{\omega \cdot 2\}$ in $E_{3}=\omega \cdot 2+1$. Then $A_{3}$ is $\Delta\left(D_{2}\right)$-complete, and $K\left(A_{3}\right)$ is $\mathbf{D}_{2}$-complete.

(iv) Let $A_{4}=\{\omega\}$ in $E_{4}=\omega+1$. Then $A_{4}$ is $\Pi_{1}^{0}$-complete, and $K\left(A_{4}\right)$ too.

(v) Let $A_{5}=\omega$ in $E_{5}=\omega+1$. Then $A_{5}$ is $\Sigma_{1}^{0}$-complete, and $K\left(A_{5}\right)$ too.

(vi) Finally $A_{6}=\{0\}$ in $E_{6}=2$ is $\Delta_{1}^{0}$-complete, as is $K\left(A_{6}\right)$.

This lemma gives the six examples of $\sigma$-ideals we needed.

Proof. Let us just give the proof of (i); the others are proved similarly, but are easier. By Lemmas 2 and 3 , it is enough to prove that $D$ is $\Sigma_{2}^{0}$-complete. So let $A \subseteq 2^{\omega}$ be $\Sigma_{2}^{0}$, say $A=\bigcup_{n} F_{n}$, with the $F_{n}$ closed and increasing. Let $T_{n}$ be the canonical tree for $F_{n}$.

Consider the following Wadge game: I plays bit-by-bit $\alpha \in 2^{\omega}$, II plays bit-by-bit $\beta \in 2^{\omega}$, and II wins if $\alpha \in A \leftrightarrow \beta \in D$. Clearly it is enough to show that II has a winning strategy in this game, for this gives a continuous $\varphi: 2^{\omega} \rightarrow 2^{\omega}$ with $A=\varphi^{-1}(D)$. Here is a winning strategy for II: Let $\left(\beta_{n}\right)_{n \in \omega}$ be an enumeration of $D$. As long as I plays in $T_{0}$, II plays along $\beta_{0}$. If at some stage $k_{0}$, I plays outside $T_{0}$, II plays $1-\beta_{0}\left(k_{0}\right)$, and then as long as I stays in $T_{1}$, II plays along $\beta_{n_{0}}$, where $n_{0}$ 
is least such that $\beta_{n_{0}}$ starts with $\beta_{0}\left\lceil k_{0}{ }^{\wedge}\left(1-\beta_{0}\left(k_{0}\right)\right)\right.$ (that $n_{0}$ exists follows from the density of $D$ ). And so on: If I leaves $T_{1}$ at stage $k_{1}$, II plays $1-\beta_{n_{0}}\left(k_{1}\right)$ and chooses $\beta_{n_{1}}$ minimum extending $\beta_{0} \uparrow k_{0}{ }^{\wedge}\left(1-\beta_{n_{0}}\left(k_{1}\right)\right), \ldots$ This strategy is clearly winning for II.

Step 2. We want to reduce any $\Pi_{1}^{1} \sigma$-ideal to one of the six classes $\Pi_{1}^{1}, \Pi_{2}^{0}, D_{2}$, $\Pi_{1}^{0}, \Sigma_{2}^{0}$ and $\boldsymbol{\Delta}_{1}^{0}$. This is done by "reducing" it to one of the six particular examples of Lemma 4. And the way to do it is by proving "Hurewicz-type results", analogs of a theorem of Hurewicz which characterizes $\boldsymbol{\Pi}_{2}^{0}$ sets among $\boldsymbol{\Pi}_{1}^{1}$ sets.

THEOREM 5. Let $E$ be compact metric, $A \subseteq E$.

(i) (Hurewicz [6]). If $A$ is $\Pi_{1}^{1}$ and not $\Pi_{2}^{0}$, there is a copy $F$ of $2^{\omega}$ within $E$, with $F \cap A$ countable dense in $F$.

(ii) If $A$ is not $D_{2}$, there is a copy $F$ of $E_{2}=\omega^{2}+1$, with $F \cap A$ a copy of $A_{2}$.

(iii) If $A$ is not $\Pi_{1}^{0}$ nor $\Sigma_{1}^{0}$, there is a copy $F$ of $E_{3}=\omega \cdot 2+1$ within $E$, with $F \cap A$ a copy of $A_{3}$.

[One could write also three results for $\Pi_{1}^{0}, \Sigma_{1}^{0}$, and $\Delta_{1}^{0}$ - but they are trivial, and of no use.]

COROllary 6. Let I be a $\Pi_{1}^{1} \sigma$-ideal in $K(E)$.

(i) If $I$ is not $\Pi_{2}^{0}, I$ is $\Pi_{1}^{1}$-complete.

(ii) If I is $\Pi_{2}^{0}$ but not $\boldsymbol{D}_{2}, I$ is $\Pi_{2}^{0}$-complete.

(iii) If $I$ is $D_{2}$ but neither $\Pi_{1}^{0}$ nor $\Sigma_{1}^{0}, I$ is $D_{2}$-complete.

Proof. We will prove (i), using Hurewicz's Theorem, 5(i). Parts (ii) and (iii) are analogous, using 5(ii) and 5(iii).

So let $I$ be $\Pi_{1}^{1}$ and not $\Pi_{2}^{0}$ in $K(E)$. Applying 5(i), we get a continuous $\varphi: 2^{\omega} \rightarrow K(E)$ and a dense countable set $D$ in $2^{\omega}$ with $\varphi^{-1}(I)=D$. Define $f: K\left(2^{\omega}\right)$ $\rightarrow K(E)$ by $f(K)=\bigcup \varphi^{\prime \prime} K$. As a composition of continuous functions, $f$ is continuous. But as $I$ is a $\sigma$-ideal, one easily checks that $K(D)=f^{-1}(I)$. As $K(D)$ is $\Pi_{1}^{1}$-complete (Lemma 4), so is $I$.

It remains to prove Theorem 5. Again parts (ii) and (iii) are easy, and we will concentrate on 5(i), i.e. Hurewicz's Theorem. It is a consequence of the following stronger form of it.

THEOREM 7 (Kechris, Louveau and Woodin [23]). Let $A_{0}$ and $A_{1}$ be two disjoint sets in $E$, with $A_{0} \in \Sigma_{1}^{1}$. Assume that no $\Sigma_{2}^{0}$ set $C$ separates $A_{0}$ from $A_{1}$ (i.e. $A_{0} \subseteq C$ and $\left.C \cap A_{1}=\varnothing\right)$. Then there exists a copy $F$ of $2^{\omega}$ within $A_{0} \cup A_{1}$, with $A_{1} \cap F$ countable dense in $F$.

[5(i) corresponds to the particular case $A_{0}=E \backslash A, A_{1}=A$.]

ProOF. First pick a continuous surjection $\varphi: 2^{\omega} \rightarrow E$, and let $B_{0}=\varphi^{-1}\left(A_{0}\right)$ and $B_{1}=\varphi^{-1}\left(A_{1}\right)$. Note that $B_{0}$ and $B_{1}$ satisfy the same hypotheses, for $B_{0}$ is $\Sigma_{1}^{1}$, and if $C$ was a $\Sigma_{2}^{0}$ set separating $B_{0}$ from $B_{1}, \varphi^{\prime \prime} C$ would be a $\Sigma_{2}^{0}$ set separating $A_{0}$ from $A_{1}$. Let $P \subseteq 2^{\omega} \times 2^{\omega}$ be $\Pi_{2}^{0}$ with $\pi P=B_{0}$, and consider

$$
P_{0}=P \backslash \bigcup\left\{V \text { open in } 2^{\omega} \times 2^{\omega}: \pi(P \cap V) \text { is } \Sigma_{2}^{0} \text {-separable from } B_{1}\right\} \text {. }
$$

Then $P_{0}$ is $\Pi_{2}^{0}$, and nonempty (otherwise $B_{0}$ would be $\Sigma_{2}^{0}$-separable from $B_{1}$ ). Moreover if $V$ is open in $2^{\omega} \times 2^{\omega}$ and $P_{0} \cap V \neq \varnothing$, then $\overline{\pi\left(P_{0} \cap V\right)} \cap B_{1} \neq \varnothing$ (otherwise $\pi(P \cap V)$ would be $\Sigma_{2}^{0}$-separable from $B_{1}$, contradicting $\left.P_{0} \cap V \neq \varnothing\right)$. Pick a basis $V_{n}$ of the topology of $2^{\omega} \times 2^{\omega}$, and for each $n$ with $P_{0} \cap V_{n} \neq \varnothing$, pick $x_{n}$ in $\overline{\pi\left(P_{0} \cap V_{n}\right)} \cap B_{1}$. Let $P_{1}=\left\{\left(x_{n}, \alpha\right): P_{0} \cap V_{n} \neq \varnothing\right.$ and $\left.\alpha \in 2^{\omega}\right\} . P_{1}$ is $\Sigma_{2}^{0}$ in $2^{\omega} \times 2^{\omega}$, 
$P_{0}$ and $P_{1}$ are disjoint, and we claim that $P_{0}$ is not $\Sigma_{2}^{0}$ separable from $P_{1}$. For otherwise, by the Baire Category Theorem, there would be an $n$ with $P_{0} \cap V_{n} \neq \varnothing$ and $P_{0} \cap V_{n} \Pi_{1}^{0}$-separable from $P_{1}$; hence $\overline{\pi\left(P_{0} \cap V_{n}\right)} \cap \pi P_{1}=\varnothing$. But $x_{n}$ is in it.

Finally, consider the following Wadge-type game: I plays bit-by-bit $\varepsilon \in 2^{\omega}$, II plays bit-by-bit $(\alpha, \beta) \in 2^{\omega} \times 2^{\omega}$, and II wins if $\varepsilon$ is eventually 0 and $(\alpha, \beta) \in P_{1}$ or $\varepsilon$ is not eventually 0 and $(\alpha, \beta) \in P_{0}$.

Note that this game is a Boolean combination of $\Pi_{2}^{0}$ and $\Sigma_{2}^{0}$ sets, hence is determined. If now I has a winning strategy in this game, this gives a continuous function $f: 2^{\omega} \times 2^{\omega} \rightarrow 2^{\omega}$ with $C=f^{-1}$ (eventually $0 \varepsilon^{\prime}$ s) a $\Sigma_{2}^{0}$ set separating $P_{0}$ from $P_{1}$. So player II has a winning strategy in this game, which gives a continuous $g: 2^{\omega} \rightarrow 2^{\omega} \times 2^{\omega}$. Consider $h=\varphi \circ \pi \circ g: 2^{\omega} \rightarrow E$. It is continuous, and if $\varepsilon$ is eventually $0, h(\varepsilon) \in A_{1}$; if $\varepsilon$ is not, $h(\varepsilon) \in A_{0}$. So if $F=h^{\prime \prime} 2^{\omega}, F$ is a closed set with $F \cap A_{0}$ and $F \cap A_{1}$ dense in $F$; so $F$ is perfect, and $F \cap A_{1}$ is countable dense in $F$. To replace $F$ by a copy of $2^{\omega}$, one just performs a Cantor type construction within $F$.

Corollary 8. Let I be a $\Pi_{1}^{1} \sigma$-ideal in $K(E)$, and $B$ a hereditary subset of I. If no $\Pi_{2}^{0}$ set separates $B$ from $K(E) \backslash I$, then any set $C$ with $B_{\sigma} \subseteq C \subseteq I$, where $B_{\sigma}$ is the $\sigma$-ideal generated by $B$, must be $\Pi_{1}^{1}$-hard.

[Corollary $6(i)$ is the particular case $B=I$.]

The proof is analogous to 6(i), using Theorem 7 instead of Theorem 5(i).

Lecture III. Bases for $\Pi_{1}^{1} \sigma$-ideals and natural $\Pi_{1}^{1}$-ranks. In this lecture, we want to continue the study of the complicated $\Pi_{1}^{1} \sigma$-ideals, i.e. those which are $\Pi_{1}^{1}$-complete.

A good example of such a $\sigma$-ideal is $K_{\omega}(E)$, the $\sigma$-ideal of countable closed subsets of $E$, when $E$ is uncountable.

[One can see it is $\Pi_{1}^{1}$-complete as follows. First we can assume $E=2^{\omega}$, as $2^{\omega}$ continuously embeds in $E$. Now $K_{\omega}\left(2^{\omega}\right)$ is dense in $K\left(2^{\omega}\right)$, as is the set $P$ of perfect compact sets. But easily $P$ is $\Pi_{2}^{0}$, and $P \cap K_{\omega}\left(2^{\omega}\right)=\{\varnothing\}$, so by the Baire Category Theorem, $K_{\omega}\left(2^{\omega}\right)$ cannot be $\Pi_{2}^{0}$. So by the dichotomy theorem, it is $\Pi_{1}^{1}$-complete. One can also give a direct reduction of $K(D)$ to $K_{\omega}\left(2^{\omega}\right)$, where $D=\left\{\alpha \in 2^{\omega}: \alpha\right.$ is eventually 0$\}$, which is $\Pi_{1}^{1}$-complete: Just define, for $K \subseteq 2^{\omega}, f(K)=\{\alpha: \exists \beta \in K \forall n(\alpha(n) \leq$ $\beta(n))\}$. Then $f$ is continuous and $K(D)=f^{-1}\left(K_{\omega}\left(2^{\omega}\right)\right)$.]

Although it is complicated, $K_{\omega}(E)$ has two nice properties:

(i) There is a simple (in fact $\Pi_{1}^{0}$ ) family of sets which generates it as a $\sigma$-ideal, the family of singletons.

(ii) One can define a derivation associated to it, the Cantor-Bendixson derivation, obtained by removing from a compact set $K$ its isolated points. Iterating the process transfinitely, one reaches at a countable step the perfect kernel of $K$. And $K$ is countable iff the perfect kernel is empty. So one gets a "semi-Borel" test for membership in $K_{\omega}(E)$.

This is the situation we want to study in general.

Definition. A basis for a $\sigma$-ideal $I$ is a hereditary subset $B$ of $I$ such that $I=B_{\sigma}=$ the $\sigma$-ideal of closed sets generated by $B$.

Clearly any $\Pi_{1}^{1} \sigma$-ideal $I$ admits a $\Pi_{1}^{1}$ basis, namely $I$ itself. The converse is also true. To see this, say that $K$ is $B$-perfect, for a hereditary family $B \subseteq K(E)$, if for any 
open $V$ with $K \cap V \neq \varnothing, \overline{K \cap V} \notin B$ (if $B=$ \{singletons $\}$, this is the usual notion of perfect sets). One easily checks, using the Baire Category Theorem, that any set $K$ contains a largest $B$-perfect subset, namely

$$
N(K)=K \backslash \bigcup\left\{V \text { open: } \overline{K \cap V} \in B_{\sigma}\right\},
$$

and that $K \backslash N(K)$ is covered by a countable union of sets in $B$.

So we get

$$
K \in B_{\sigma} \Leftrightarrow N(K)=\varnothing
$$

Lemma 1. If $B$ is a hereditary $\Pi_{1}^{1}$ family, the $\sigma$-ideal $B_{\sigma}$ it generates is also $\boldsymbol{\Pi}_{1}^{1}$.

Proof. If $\left(V_{n}\right)$ is a basis of the topology of $E$, one has

$$
K \text { is } B \text {-perfect } \Leftrightarrow \forall n\left(\overline{K \cap V_{n}} \notin B\right),
$$

so that $B$-perfect is $\Sigma_{1}^{1}$ if $B$ is $\Pi_{1}^{1}$.

Moreover

$$
K \in B_{\sigma} \Leftrightarrow \forall L[(L \neq \varnothing \text { and } L \subseteq K) \Rightarrow L \text { is not } B \text {-perfect }]
$$

and so $B_{\sigma}$ is $\Pi_{1}^{1}$.

Can one do better, i.e. is it possible for a $\Pi_{1}^{1} \sigma$-ideal to have a Borel basis? The answer is clearly yes for $\Pi_{2}^{0} \sigma$-ideals, but $K_{\omega}\left(2^{\omega}\right)$ is an example of a $\Pi_{1}^{1}$-complete $\sigma$-ideal with a $\boldsymbol{\Pi}_{1}^{0}$ basis. On the other hand, if $A$ is $\Pi_{1}^{1}$ not Borel, $K(A)$ is a $\Pi_{1}^{1} \sigma$ ideal with no Borel basis [for one has, for any basis $B, x \in A \leftrightarrow\{x\} \in B$ ].

THEOREM 2. The following are equivalent, for a $\Pi_{1}^{1} \sigma$-ideal $I$ :

(i) I admits a Borel basis.

(ii) I admits a $\Sigma_{1}^{1}$ basis.

(iii) I-perfect is Borel.

Proof. Clearly (i) $\Rightarrow$ (ii). We prove next that (ii) $\Rightarrow$ (iii). If $B$ is a $\Sigma_{1}^{1}$ basis, one has

$B$-perfect $=I$-perfect

so that, as $I$ is $\Pi_{1}^{1}$ and $B$ is $\Sigma_{1}^{1}, B$-perfect is both $\Sigma_{1}^{1}$ and $\Pi_{1}^{1}$, hence is Borel.

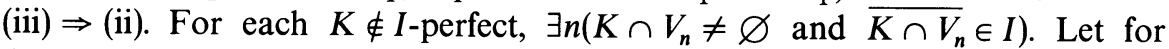
each $n$

$$
C_{n}=\left\{K: K \cap V_{n} \neq \varnothing \text { and }{\overline{K \cap V_{n}}}_{i} I\right\} .
$$

As $I$ is $\Pi_{1}^{1}$, each $C_{n}$ is $\Pi_{1}^{1}$, and the $C_{n}$ 's cover the Borel set $C=K(E) \backslash I$-perfect. By Novikov's selection theorem there is a Borel function $\varphi: C \rightarrow \omega$ such that for any $K \in C, K \cap V_{\varphi(K)} \neq \varnothing$ and $\overline{K \cap V_{\varphi(K)}} \in I$.

Let $B=\left\{L: \exists K \in C\left(L \subseteq \overline{K \cap V_{\varphi(K)}}\right)\right\}$. This is a $\Sigma_{1}^{1}$ hereditary subset of $I$. And it is a basis for $I$, for if $K \in I \backslash B_{\sigma}$, its $B_{\sigma}$-perfect kernel $L$ is nonempty and in $I$, hence in $C$. But then $L \cap V_{\varphi(L)} \neq \varnothing$ and $\overline{L \cap V_{\varphi(L)}} \in B$ by definition of $B$, i.e. $L$ is not $B_{\sigma}$-perfect, a contradiction.

Finally (ii) $\Rightarrow$ (i) using repeatedly the separation theorem: If $B_{0}$ is a $\Sigma_{1}^{1}$ basis for $I$, one can find $C_{0}$ Borel with $B_{0} \subseteq C_{0} \subseteq I$. Its hereditary closure $B_{1}$ is $\Sigma_{1}^{1}$ with $C_{0} \subseteq B_{1} \subseteq I$. So we can find $C_{1}$ Borel with $B_{1} \subseteq C_{1} \subseteq I$, and so on. Doing this $\omega$ times, one gets sequences $B_{n}$ of $\Sigma_{1}^{1}$ hereditary sets and $C_{n}$ of Borel sets with $B_{n} \subseteq C_{n} \subseteq B_{n+1} \subseteq I$, and $B=\bigcup_{n} B_{n}=\bigcup_{n} C_{n}$ is a Borel basis for $I$. 
A $\boldsymbol{\Pi}_{1}^{1}$-rank (or $\boldsymbol{\Pi}_{1}^{1}$-norm) on a $\boldsymbol{\Pi}_{1}^{1}$ set $A$ is a function $\varphi: A \rightarrow \omega_{1}$ with the property that the relations

$$
x \in A \text { and }(y \notin A \text { or } \varphi(x) \leq \varphi(y))
$$

and

$$
x \in A \text { and }(y \notin A \text { or } \varphi(x)<\varphi(y))
$$

are both $\Pi_{1}^{1}$.

Any $\Pi_{1}^{1}$ set admits a $\Pi_{1}^{1}$-rank (in fact many of them). And such a rank $\varphi$ gives a sequence of approximations

$$
A_{\xi}=\{x \in A: \varphi(x) \leq \xi\}
$$

which are increasing Borel sets.

The $\omega_{1}$-sequence $A_{\xi}$ is a strict hierarchy iff $A$ is not Borel. Moreover, it satisfies the boundedness theorem: any $\Sigma_{1}^{1}$ subset $B$ of $A$ is already a subset of some $A_{\xi}$.

So in particular $\Pi_{1}^{1} \sigma$-ideals admit $\Pi_{1}^{1}$-ranks. But what we are looking for are "natural" ranks, reflecting the structure of the $\sigma$-ideal. The next result shows that this is possible when $I$ admits a Borel basis.

If $B$ is a hereditary family in $K(E)$, define the $B$-derivation $d_{B}$ by

$$
d_{B}(K)=K \backslash \bigcup\{V \text { open: } \overline{K \cap V} \in B\} \text {. }
$$

Iterating the derivation, define $K_{B}^{0}=K, K_{B}^{\alpha+1}=d_{B}\left(K_{B}^{\alpha}\right)$ and $K_{B}^{\lambda}=\bigcap_{\alpha<\lambda} K_{B}^{\alpha}$ for limit $\lambda$.

For each $K$, the sequence $K_{B}^{\alpha}$ decreases, hence the process stops at some ordinal $\alpha(K)<\omega_{1}$. Clearly $K_{B}^{\alpha(K)}$ is the $B$-perfect kernel of $K$, so that

$$
K_{B}^{\alpha(K)}=\varnothing \Leftrightarrow K \in B_{\sigma}
$$

and we can define the $B$-rank $\mathrm{rk}_{B}(K)$, for $K \in B_{\sigma}$, as $\mathrm{rk}_{B}(K)=$ least $\alpha\left(K_{B}^{\alpha}=\varnothing\right)$. [For the usual Cantor-Bendixson derivation, this is the Cantor-Bendixson rank of countable closed sets.]

THEOREM 3. Suppose I is a $\Pi_{1}^{1} \sigma$-ideal with Borel basis $B$. Then the rank $\mathrm{rk}_{B}$ is a $\Pi_{1}^{1}$-rank on $I$.

The proof is a rather tedious computation.

Let $\mathbb{Q}^{+}$be the nonnegative rationals, and define

$$
\begin{aligned}
L O & =\left\{R \subseteq \mathbb{Q}^{+}: 0 \in R\right\}, \\
W O & =\{R \in L O: R \text { is well ordered by the usual ordering of } \mathbb{Q}\} .
\end{aligned}
$$

For $R \in W O$, let $\operatorname{lh}(R)$ be its associated ordinal and for $p \in R$

$$
\operatorname{lh}(p, R)=\operatorname{lh}(R \cap[0, p[)
$$

so that

$$
\operatorname{lh}(R)=\sup _{p \in R}(\operatorname{lh}(p, R)+1)
$$

We make three claims:

(a) The relation $K \notin I \vee\left(R \in W O \wedge \operatorname{lh}(R) \leq \mathrm{rk}_{B}(K)\right)$ is $\Sigma_{1}^{1}\left(\right.$ in $\left.K(E) \times 2^{\mathbf{Q}^{+}}\right)$.

(b) The relation $K \notin I \vee\left(R \in W O \wedge \operatorname{lh}(R)<\mathrm{rk}_{B}(K)\right)$ is $\Sigma_{1}^{1}$.

(c) The relation $R \in W O \wedge\left(L \notin I \vee \operatorname{lh}(R)<\operatorname{rk}_{B}(L)\right)$ is $\Pi_{1}^{1}$. 
One easily gets the result from the claim, because

(*)

$$
\begin{aligned}
& K \in I \wedge\left[L \notin I \vee \mathrm{rk}_{B}(K) \leq \mathrm{rk}_{B}(L)\right] \\
& \Leftrightarrow K \in I \wedge \forall R\left\{\left[K \notin I \vee\left(R \in W O \wedge \mathrm{lh}(R)<\mathrm{rk}_{B}(K)\right)\right]\right. \\
& \left.\quad \Rightarrow\left[R \in W O \wedge\left(L \notin I \vee \operatorname{lh}(R)<\mathrm{rk}_{B}(L)\right)\right]\right\} .
\end{aligned}
$$

Using (b) and (c), (*) is then $\Pi_{1}^{1}$.

Similarly,

$$
\begin{aligned}
& K \in I \wedge\left[L \notin I \vee \mathrm{rk}_{B}(K)<\mathrm{rk}_{B}(L)\right] \\
& \Leftrightarrow K \in I \wedge \forall R\left\{\left[K \notin I \vee\left(R \in W O \wedge \mathrm{lh}(R) \leq \mathrm{rk}_{B}(K)\right)\right]\right. \\
& \quad\left.\Rightarrow\left[R \in W O \wedge\left(L \notin I \vee \operatorname{lh}(R)<\mathrm{rk}_{B}(L)\right)\right]\right\}
\end{aligned}
$$

which by (a) and (c) is also $\Pi_{1}^{1}$.

Let us prove (a) ((b is similar). Consider the relation

$$
\left.\begin{array}{rl}
S(R, K) \Leftrightarrow \exists h \in K(E)^{\mathbf{Q}^{+}}\left[h(0)=K \wedge \forall p \in R, p>0\left(h(p)=\bigcap_{\substack{q \in R \\
q<p}} d_{B}(h(q))\right.\right. \\
\wedge \forall p \in R(h(p) \neq \varnothing)
\end{array}\right) .
$$

As $B$ is Borel, the relation $S$ is $\Sigma_{1}^{1}$. So to get (a), it is enough to prove that

$$
K \notin I \vee\left(R \in W O \wedge \operatorname{lh}(R) \leq \mathrm{rk}_{B}(K)\right) \Leftrightarrow K \notin I \vee S(R, K) .
$$

The direction $\Rightarrow$ is easy: If $K \in I$ and $R \in W O$ and $\operatorname{lh}(R) \leq \operatorname{rk}_{B}(K)$, the function $h$ defined by

witnesses $S(R, K)$.

$$
h(p)= \begin{cases}E & \text { if } p \notin R, \\ K_{B}^{\operatorname{lh}(p, R)} & \text { if } p \in R\end{cases}
$$

Conversely, suppose $K \in I$, and let $h$ witness $S(R, K)$. For $p \in R$, let $f(p)=$ least $\beta\left(h(p) \nsubseteq K_{B}^{\beta+1}\right)$. Note that as $h(p) \neq \varnothing$ but $K_{B}^{\mathrm{rk}_{B}(K)}=\varnothing, f(p)<\operatorname{rk}_{B}(K)$.

Now by definition of $f(p), h(p) \subseteq K_{B}^{f(p)}$. So if $p<r$ in $R$, one has

$$
h(r)=\bigcap_{\substack{q<r \\ q \in R}} d_{B}(h(q)) \subseteq d_{B}(h(p)) \subseteq d_{B}\left(K_{B}^{f(p)}\right)=K_{B}^{f(p)+1},
$$

and hence $f(p)+1 \leq f(r)$.

This shows that $f$ is strictly increasing on $R$, hence $R \in W O$. And by induction on $R$, one must have $h(p)=K_{B}^{\mathrm{lh}(p, R)} \neq \varnothing$ for all $p \in R$, so $\operatorname{lh}(R) \leq \mathrm{rk}_{B}(K)$.

The proof of (c) is a bit easier. Define

$$
\begin{aligned}
& T(R, L) \Leftrightarrow \exists h \in K(E)^{\mathbb{Q}^{+}}\left[\begin{array}{l}
h(0)=L \wedge \forall p \in R, p>0\left(h(p)=\bigcap_{\substack{q \in R \\
q<p}} d_{B}(h(q))\right. \\
\wedge \exists p \in R\left(d_{B}(h(p))=\varnothing\right)
\end{array}\right] . \\
& \text { Again } T \text { is } \Sigma_{1}^{1} \text { and (c) follows from the equivalence }
\end{aligned}
$$

$$
R \in W O \wedge\left(L \notin I \vee \operatorname{lh}(R)<\operatorname{rk}_{B}(L)\right) \Leftrightarrow R \in W O \wedge \neg T(R, L) .
$$

Direction $\Rightarrow$. Assume $R \in W O$ but $T(R, L)$. Then if $h$ witnesses it, one easily proves by induction on $R$ that $h(p)=L_{B}^{\ln (p, R)}$. So there is a $p \in R$ with $L_{B}^{\ln (p, R)+1}=$ $\varnothing$, so $L \in I$ and $\operatorname{rk}_{B}(L) \leq \operatorname{lh}(R)$. 
Direction $\Leftarrow$. Assume $R \in W O$, but $L \in I$ and $\operatorname{rk}_{B}(L) \leq \operatorname{lh}(R)$. Then $h$ defined by

$$
h(p)= \begin{cases}E & \text { if } p \notin R, \\ L_{B}^{\operatorname{lh}(p, R)} & \text { if } p \in R\end{cases}
$$

witnesses $T(R, L)$, because $\operatorname{rk}_{B}(L)$ is always a successor ordinal, hence there is a $p \in R$ with $\operatorname{rk}_{B}(L)=\operatorname{lh}(p, R)+1$, and for this $p, d_{B}(h(p))=\varnothing$.

Lecture IV. Extensions of $\sigma$-ideals of closed sets and the basis theorem. Let $I$ be a $\sigma$-ideal of closed sets. The most immediate way to extend $I$ to a $\sigma$-ideal of arbitrary sets is the exterior method.

Let

$$
I_{\text {ext }}=\left\{A \subseteq E: \exists\left(K_{n}\right)_{n \in \omega} \text { in } I\left(A \subseteq \bigcup_{n} K_{n}\right)\right\} .
$$

Then $I_{\text {ext }}$ is a $\sigma$-ideal of sets, and $I_{\text {ext }} \cap K(E)=I$. Moreover, $I_{\text {ext }}$ is clearly the smallest $\sigma$-ideal of sets extending $I$.

For a typical example consider $I_{m}$, the $\sigma$-ideal of nowhere dense closed sets: Its exterior extension is the $\sigma$-ideal of meager sets. Similarly the exterior extension of $K_{\omega}(E)$ is the $\sigma$-ideal of countable sets. However it may happen that the exterior extension is not the natural one. For example, if $\lambda$ is the Lebesgue measure on $2^{\omega}$, the exterior extension of $I_{\lambda}=\{K: \lambda(K)=0\}$ is not the $\sigma$-ideal of sets of Lebesgue measure 0 - for there are dense $G_{\delta}$ 's of measure 0 , whereas any set in $\left(I_{\lambda}\right)_{\text {ext }}$ is meager. This example suggests another extension, the one from the interior.

Let

$$
I_{\mathrm{int}}=\{A \subseteq E: K(A) \subseteq I\} .
$$

Clearly $I_{\text {int }}$ is hereditary, $I_{\text {ext }} \subseteq I_{\text {int }}$ and $I_{\text {int }} \cap K(E)=I$. But in general $I_{\text {int }}$ is not even an ideal: For example, for $I=I_{m}, I_{\text {int }}$ consists of the sets $A$ with $E \backslash A$ dense in $E$.

Definition. A $\sigma$-ideal $I$ of closed sets is calibrated if for any closed set $K$, if there is a sequence $\left(K_{n}\right)$ in $I$ such that $K \backslash \bigcup_{n} K_{n} \in I_{\text {int }}$, then $K \in I$.

The $\sigma$-ideal $I_{m}$ is not calibrated. On the other hand, $K_{\omega}(E), I_{\mu}, I_{\gamma}$ for a subadditive capacity $\gamma, J_{\gamma}, I_{\mu^{h}}$ for a Hausdorff measure $\mu^{h}, J_{\mu^{h}}$, and, as we will see, $U$ and $U_{0}$ are all calibrated $\sigma$-ideals. The terminology comes from analogous "interior approximation" notions in capacity theory introduced by Dellacherie.

Proposition 1. Let I be a $\sigma$-ideal of closed sets. The following are equivalent:

(i) I is calibrated.

(ii) $I_{\text {int }} \cap G_{\delta}$ is a $\sigma$-ideal of $G_{\delta}$ sets.

(iii) $I_{\mathrm{int}} \cap G_{\delta \sigma}$ is a $\sigma$-ideal of $G_{\delta \sigma}$ sets.

Proof. Clearly (iii) $\Rightarrow$ (ii) $\Rightarrow$ (i). So assume $I$ is calibrated, and let $\left(H_{n}\right)$ be a sequence of $G_{\delta \sigma}$ sets in $I_{\text {int }}$, towards showing $H=\bigcup_{n} H_{n}$ is in $I_{\text {int }}$. Clearly without loss of generality we may assume the $H_{n}$ 's are $G_{\delta}$. So towards a contradiction, let $K \subseteq \bigcup_{n} H_{n}, K \notin I$. Write $K \backslash H_{0}=\bigcup_{n} K_{n}^{0}, K_{n}^{0}$ compact. Using the calibration property, we get $n_{0}$ such that $K_{n_{0}}^{0} \notin I$. Write $K_{n_{0}}^{0} \backslash H_{1}=\bigcup_{n} K_{n}^{1}$ with $K_{n}^{1}$ compact. Again there is $n_{1}$ with $K_{n_{1}}^{1} \notin I$. Continuing this way, we get a decreasing sequence $K_{n_{k}}^{k}$ of compact subsets of $K$ with $K_{n_{k}}^{k} \notin I$ and $K_{n_{k}}^{k} \cap H_{k}=\varnothing$. But then in particular $\bigcap_{k} K_{n_{k}}^{k} \neq \varnothing$, and is disjoint from $\bigcup_{k} H_{k}$, contradicting that $K \subseteq \bigcup_{n} H_{n}$. 
The next result is the key for establishing an important interplay between descriptive set theoretic and structural properties of $\sigma$-ideals of closed sets. It relates, for calibrated $\sigma$-ideals, the existence of nontrivial bases with the category of the sets in $I_{\text {int }}$.

THEOREM 2 (The basis theorem, Kechris, Louveau and Woodin [23]). Let I be a calibrated $\sigma$-ideal in $K(E)$. Assume that $I$ admits a nontrivial basis, i.e. a basis $B$ such that for every open $V \neq \varnothing$ in $E, B \cap K(V) \neq I \cap K(V)$. Then every $G_{\delta}$ (hence every Borel) set in $I_{\mathrm{int}}$ is meager.

Proof. It is clearly enough to show that if $H \subseteq E$ is a dense $G_{\delta}, H$ contains a compact set which is not in $I$ (by relativizing the argument to an open set). And we may assume that $\forall x \in H(\{x\} \in I)$; otherwise the conclusion is trivially true. Also the existence of a nontrivial basis implies that any compact set in $I$ is meager, for otherwise there is a nonempty $V$ with $\bar{V} \in I$, hence by the Baire Category Theorem a nonempty open $V^{\prime}$ with $\bar{V}^{\prime} \in B$, and, on $V^{\prime}, B \cap K\left(V^{\prime}\right)=I \cap K\left(V^{\prime}\right)$.

Now if $K$ is meager in $E$ and $V$ is open with $K \subseteq V$, one can pick a countable set of points $D(K, V)$ in $(H \cap V) \backslash K$, with $\overline{D(K, V)}=D(K, V) \cup K$.

Write $H=\bigcap_{n} \Omega_{n}$, with $\Omega_{n}$ dense open in $E$. We construct inductively sets $\left(K_{s}\right)_{s \in \omega<\omega}$ as follows:

By the hypothesis, $\Omega_{0}$ contains a compact set $K_{\varnothing} \in I \backslash B$. Write $D\left(K_{\varnothing}, \Omega_{0}\right)$ as $\left\{x_{n}: n \in \omega\right\}$. Choose open sets $U_{n} \ni x_{n}$, small enough so that $\bar{U}_{n} \subseteq \Omega_{1}, \bar{U}_{n} \cap \bar{U}_{m}=$ $\varnothing$ for $n \neq m$, and $\bar{U}_{n} \cap K_{\varnothing}=\varnothing$. Then let $K_{n}$ be a compact subset of $U_{n}$ with $K_{n} \in I \backslash B$. Suppose the construction of the $K_{s}$ and $U_{s}$ has been done for $s \in \omega^{n}$, and write $D\left(K_{\underline{s}}, U_{s}\right)=\left\{x_{s^{n} n}: n \in \omega\right\}$. Choose $U_{s^{n} n} \ni x_{s^{n} n}$ small enough so that $\bar{U}_{s^{n} n}$ $\subseteq \Omega_{n+1} \cap U_{s}, \bar{U}_{s^{\prime} n} \cap \bar{U}_{s^{\prime} m}=\varnothing$ for $m \neq n$, and $\bar{U}_{s^{\prime} n} \cap K_{s}=\varnothing$. Then choose $K_{s^{\prime} n} \subseteq$ $U_{s^{\prime} n}, K_{s^{\prime} n} \in I \backslash B$.

Now let $K=\bigcap_{n}\left(\overline{\bigcup_{s \in \omega^{n}} K_{s}}\right)$. This is a compact subset of $E$. We claim first that $K \subseteq H \cup \bigcup_{s \in \omega<\omega} K_{s}$. To see this, let $x \in K \backslash \bigcup_{s \in \omega<\omega} K_{s}$. So in particular $x \notin K_{\varnothing}$. But as $K \subseteq \overline{\bigcup_{n} K_{n}} \subseteq \overline{\bigcup_{n} \bar{U}_{n}}=\bigcup_{n} \bar{U}_{n} \cup K_{\varnothing}$, it follows that for some $n_{0}, x \in \bar{U}_{n_{0}}$. But then $x \in \bar{U}_{n_{0}} \cap K \subseteq \overline{\bigcup_{n \in \omega} K_{n_{0} n}} \subseteq \overline{\bigcup_{n} \bar{U}_{n_{0} n}}=\bigcup_{n} \overline{U_{n_{0} n}} \cup K_{n_{0}}$. And as $x \notin K_{n_{0}}$, there is $n_{1}$ such that $x \in \overline{U_{n_{0} n_{1}}}$. Continuing this way, one gets $\alpha \in \omega^{\omega}$ such that, for all $k, x \in \overline{U_{\alpha \mid k}} \subseteq \Omega_{k}$, hence $x \in H$.

Secondly we claim that $K \notin I$. Otherwise, by the Baire Category Theorem, there is an open $V$ with $K \cap V \neq \varnothing$ and $\overline{K \cap V} \in B$, as $B$ is a basis for $I$.

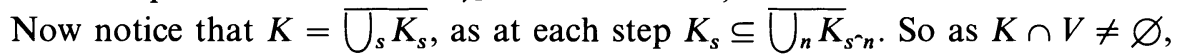
there is an such that $K_{s} \cap V \neq \varnothing$. But then for some $n, \overline{U_{s^{\prime} n}} \subseteq V$, so $K_{s^{\prime} n} \subseteq K \cap V$. But $K_{s^{\prime} n} \notin B$, contradicting that $\overline{K \cap V} \in B$.

So we can apply the calibration property of $I$ : As $K \notin I$ but for all $s K_{s} \in I$, $K \backslash \bigcup_{s} K_{s}$ is not in $I_{\text {int }}$; hence a fortiori $H$ is not in $I_{\text {int }}$.

This theorem has many applications. For example its conclusion fails for $I_{\lambda}$, as there is a dense $G_{\delta}$ of Lebesgue measure 0 . So $I_{\lambda}$ admits no nontrivial basis. More generally,

COROLlARY 3. Let $\gamma$ be a measure, or a Hausdorff measure, or a subadditive capacity on $E$ which is continuous (i.e. such that $\gamma(K)=0 \Rightarrow \gamma(K \cup\{x\})=0$ for all $x \in E$ ). Let $I$ be a $\sigma$-ideal of closed sets which is calibrated and admits a nontrivial basis. Then there is a closed set $K$ with $\gamma(K)=0$ but $K \notin I$. 
Proof. In any of the above cases, one can build a dense $G_{\delta}$-set $H$ with $\gamma(H)=0$. One then uses Theorem 3 to get $K$ inside $H$.

Another application (that we won't prove) is.

Corollary 4. Let $A$ be $a \Pi_{1}^{1}$ subset of $E$. Then $K(A)$ has a Borel basis iff $A$ is the difference of two $\Pi_{2}^{0}$ sets.

But the main application is

Theorem 5 (Debs and Saint Raymond [4]). Let I be a $\Pi_{1}^{1} \sigma$-ideal in $K(E)$. Assume that the following conditions hold:

(i) I is calibrated.

(ii) If $L$ is a nonempty $I$-perfect set, $I \cap K(L)$ is $\Pi_{1}^{1}$-complete.

(iii) I admits a Borel basis.

Then

$$
I_{\text {int }} \cap \Sigma_{1}^{1}=I_{\text {ext }} \cap \Sigma_{1}^{1} .
$$

The conclusion of Theorem 6 is extremely strong. In fact among our examples of $\sigma$-ideals, the only ones for which it is known to hold are $K_{\omega}(E)$ and, as we will see later, $U_{0}$.

Proof. Fix a Borel basis $B$ for $I$. Let $A$ be a $\Sigma_{1}^{1}$ subset of $E$ not in $I_{\text {ext }}$, towards showing $A \notin I_{\text {int }}$. Let $H \subseteq E \times 2^{\omega}$ be a $\Pi_{2}^{0}$ set with $A=\pi H$, and let

$$
H^{\prime}=H \backslash \bigcup\left\{V \text { open in } E \times 2^{\omega}: \pi(V \cap H) \in I\right\} .
$$

As $A \notin I_{\text {ext }}, H^{\prime} \neq \varnothing$. Let $F=\overline{H^{\prime}} . F$ is a compact metric space, and we define a new $\sigma$-ideal $J \subseteq K(F)$ by $K \in J \Leftrightarrow \pi K \in I$.

We claim that $J$ satisfies the hypotheses of the basis theorem, i.e. is calibrated and has a nontrivial basis. This will finish the proof, for then by the basis theorem $H^{\prime} \notin J_{\text {int }}$, so contains a compact set $K$ with $\pi K \notin I$. But $\pi K \subseteq A$, as desired.

To see that $J$ is calibrated, let $K \subseteq F$ and $\left(K_{n}\right)$ in $J$ be such that $K \backslash \bigcup_{n} K_{n} \in J_{\text {int }}$. Consider $\pi K \backslash \bigcup_{n} \pi K_{n}$. As $\pi K_{n} \in I$ for all $n$, if $\pi K \notin I$ then $\pi K \backslash \bigcup_{n} \pi K_{n}$ contains a compact $L \notin I$, by calibration of $I$. But then $K \cap \pi^{-1} L \subseteq K \backslash \bigcup_{n} K_{n}$ is not in $J$, a contradiction. So $\pi K \in I$, and $K \in J$.

To see that $J$ admits a nontrivial basis, consider $B^{*}=\{K \subseteq F: \pi K \in B\}$. Clearly $B^{*}$ is a basis for $J$. Now if $V$ is a nonempty open subset in $F$, then by definition of $H^{\prime}, \pi\left(V \cap H^{\prime}\right) \notin I_{\text {ext }}$, so $\overline{\pi V}=\overline{\pi(V \cap H)} \notin I$. Applying this to all open subsets of $V$, we get that $L=\overline{\pi V}$ is a nonempty $I$-perfect set. But then $K(L) \cap I$ is $\Pi_{1}^{1}$-complete, and $K(L) \cap B$ is Borel. So they are different, and hence $J$ and $B^{*}$ are different on $\bar{V}$. As this is true for all nonempty $V, B^{*}$ is a nontrivial basis for $J$.

Note that this theorem can be used both ways, either to infer structural properties from the existence of a Borel basis, or to prove the nonexistence of a Borel basis from structural properties. We will see applications of it both ways for the $\sigma$-ideals $U$ and $U_{0}$.

Lecture V. Elements of the classical theory of sets of uniqueness. The goal of this lecture is to discuss some basic facts about sets of uniqueness and achieve some familiarity with this notion. Some of the highlights are the Cantor uniqueness theorem, Rajchman's examples of perfect sets of uniqueness and Bary's theorem on countable unions of closed sets of uniqueness. (The standard references here are Bary [2] and Zygmund [37].)

First we introduce some notation and terminology: 
A trigonometric series is an expression of the form

$$
S \sim \sum_{n=-\infty}^{+\infty} c_{n} e^{i n x}, \quad \text { where } c_{n} \in \mathbb{C}, x \in \mathbb{R}
$$

We write

$$
\sum c_{n} e^{i n x}=s \quad \text { iff } \quad \lim _{N \rightarrow \infty} \sum_{n=-N}^{N} c_{n} e^{i n x}=s .
$$

$\mathbb{T}:=\mathbb{R} / 2 \pi \mathbb{Z}$ is the unit circle.

$\lambda$ is the normalized Lebesgue measure on $\mathbb{T}$.

$P \subseteq \mathbb{T}$ is a set of uniqueness if every trigonometric series converging to 0 off $P$ is identically 0 . Else $P$ is a set of multiplicity. We denote by $\mathscr{U}$ and $\mathscr{M}$ the corresponding classes of sets.

Proposition 1. If $P \subseteq \mathbb{T}$ is a Lebesgue measurable $\mathscr{U}$-set, then $\lambda(P)=0$.

ProOF. Else there is $F \subseteq P, F$ closed with $\lambda(F)>0$. Let $f=\chi_{F}$, the characteristic function of $F$. By standard Fourier analysis

$$
\left.S(f) \sim \sum \hat{f}(n) e^{i n x} \quad \text { (the Fourier series of } f\right)
$$

converges to 0 off $F$. Therefore $\hat{f}=0$. But $\hat{f}(0)=\lambda(F)$.

Recall here the

Category Problem. If $P$ is a Borel $\mathscr{U}$-set, is $P$ of the first category?

THEOREM 2 (Cantor, 1870). $\varnothing \in \mathscr{U}$.

The proof is based on the Riemann theory of trigonometric series. The key idea is to consider for each $S \sim \sum c_{n} e^{\text {inx }}$ with bounded $c_{n}$ (i.e. $\left.\sup \left|c_{n}\right|<\infty\right)$ the following function, called the Riemann function of $S$, obtained by integrating $\sum c_{n} e^{\text {inx }}$ formally twice:

$$
F_{S}(x):=\frac{c_{0} x^{2}}{2}-\sum_{-\infty}^{+\infty} \frac{c_{n}}{n^{2}} \cdot e^{i n x}, \quad x \in \mathbb{R}
$$

(the prime means 0 is omitted). Clearly $F_{S}$ is continuous.

Given $F: \mathbb{R} \rightarrow \mathbb{C}$, its second Schwarz derivative is given by

$$
D^{2} F(x)=\lim _{h \rightarrow 0} \frac{\Delta^{2} F(x, h)}{h^{2}}
$$

where $\Delta^{2} F(x, h)=F(x+h)+F(x-h)-2 F(x)$, if this limit exists. The key relationship of $F_{S}$ to $S$ is given by

RIEMANN's FIRST LEMMA. If $S \sim \sum c_{n} e^{i n x}$ has bounded $c_{n}$, then

$$
\sum c_{n} e^{i n x}=s \Rightarrow D^{2} F_{S}(x)=s .
$$

Proof. This lemma follows from elementary calculations using Toeplitz's theorem on regular summability methods.

The following can be also proved by elementary means.

SchWARZ's Lemma. If $F:(a, b) \rightarrow \mathbb{C}$ and $D^{2} F(x)=0$ for all $x \in(a, b)$, then $F$ is linear on $(a, b)$.

Finally we have the basic

CANTOR-LeBesgue Lemma. If $\sum c_{n} e^{i n x}=0$ on a set of positive Lebesgue measure, then $c_{n} \rightarrow 0$. 
Proof. Some trigonometry plus the Riemann-Lebesgue Lemma (if $f \in L^{1}(\mathbb{T})$, then $\hat{f}(n) \rightarrow 0$ as $|n| \rightarrow \infty)$.

PROOF OF TheOREM 2. Say $\sum c_{n} e^{i n x}=0, \forall x$. Then $c_{n} \rightarrow 0$; in particular $c_{n}$ is bounded. So $D^{2} F_{S}(x)=0, \forall x$; thus $F_{S}$ is linear, say

$$
c_{0} \frac{x^{2}}{2}-\sum^{\prime} \frac{c_{n}}{n^{2}} e^{i n x}=a x+b .
$$

Put $x=\pi$ and $x=-\pi$, and subtract to get $a=0$. Put $x=0$ and $x=2 \pi$, and subtract to get $c_{0}=0$. So $b=-\sum^{\prime} c_{n} e^{i n x} / n^{2}$, and by term-by-term integration, $c_{n}=0, \forall n \neq 0$.

Cantor (1872) essentially proved next that all countable closed sets are in $\mathscr{U}$. It was through this work that he was led to the creation of set theory. We will prove here one key step in Cantor's proof. (The full Cantor result will be a corollary of this and later results in this lecture.)

Riemann's Second Lemma. Let $S \sim \sum c_{n} e^{i n x}$ with $c_{n} \rightarrow 0$. Then $F$ is "smooth", i.e.

$$
\frac{\Delta^{2} F_{S}(x, h)}{h}=\frac{F_{S}(x+h)-F_{S}(x)}{h}-\frac{F_{S}(x)-F_{S}(x-h)}{h} \rightarrow 0
$$

as $h \rightarrow 0$ (uniformly on $x$ ).

Proof. Similar calculations to those for Riemann's First Lemma.

THEOREM 3 (Cantor, 1872). Every finite set is in $U$.

Proof. For notational simplicity take a single point $x_{0} \in \mathbb{T}$. Say $\sum c_{n} e^{i n x}=0$ for $x \neq x_{0}$. As in Theorem 2 it is enough to show that $F_{S}$ is linear in every interval of length $2 \pi$, say $(0,2 \pi)$. Again without loss of generality we can assume $x_{0} \in(0,2 \pi)$. Then $F_{S}$ is linear in $\left(0, x_{0}\right)$ and $\left(x_{0}, 2 \pi\right)$. But by Riemann's Second Lemma, $F_{S}$ cannot have a corner at $x_{0}$, so $F_{S}$ is linear in $(0,2 \pi)$.

Next we will recall the so-called Rajchman multiplication theory (1920's), which is very useful in localization arguments. (Rajchman was Zygmund's teacher.)

Let $S \sim \sum c_{n} e^{i n x}$ have bounded $c_{n}$. Let $f \in A(\mathbb{T})$, i.e. $f: \mathbb{T} \rightarrow \mathbb{C}$ is continuous and $\sum|\hat{f}(n)|<\infty$. Thus $f(x)=\sum \hat{f}(n) e^{i n x}$ uniformly. Define the formal product $S(f) \cdot S$ by

$$
S(f) \cdot S \sim \sum C_{n} e^{i n x},
$$

where $C_{n}=\sum_{k} c_{k} \hat{f}(n-k)$, the convolution of $c_{n}$ and $\hat{f}(n)$. It is easily checked that $C_{n}$ is defined and bounded.

LeMma 4. $c_{n} \rightarrow 0 \Rightarrow C_{n} \rightarrow 0$.

ProOF. Elementary calculations.

If $f$ is "nice", say $C^{\infty}$, so that it has rapidly decreasing $\hat{f}(n)$, the formal product behaves as expected.

LEMma 5. If $\varphi \in C^{\infty}(\mathbb{T})(:=$ the class of infinitely differential functions) and $S \sim \sum c_{n} e^{i n x}$ has $c_{n} \rightarrow 0$, then $S(\varphi) \cdot \sum c_{n} e^{i n x}$ and $\varphi(x) \sum c_{n} e^{i n x}$ are (uniformly) equiconvergent, i.e.

$$
\sum_{-N}^{N} C_{n} e^{i n x}-\varphi(x) \sum_{-N}^{N} c_{n} e^{i n x} \rightarrow 0
$$

as $N \rightarrow \infty$, uniformly on $x$, where $S(\varphi) \cdot \sum c_{n} e^{i n x} \sim \sum C_{n} e^{i n x}$. 
Proof. Somewhat more involved but still elementary calculations.

Our first application of the Rajchman theory will be to produce examples of perfect $\mathscr{U}$-sets.

Definition. A set $E \subseteq \mathbb{T}$ is an $H$-set if there is a nonempty interval (open $\operatorname{arc}$ ) in $T$ and $0<n_{1}<n_{2}<\cdots$ with $\left(n_{k} E\right) \cap I=\varnothing($ where $m E=\{m x(\bmod 2 \pi): x \in E\})$.

Examples include finite sets and the Cantor $1 / 3$-set $\left(n_{k}=3^{k}, I=\right.$ the middle third interval of $[0,2 \pi])$.

THEOREM 6 (Rajchman, 1921). Every $H$-set, in particular the Cantor 1/3-set, is a U-set.

Proof. The closure of an $H$-set is an $H$-set, so it is enough to work with a closed $H$-set $E$. Let $n_{k}$ and $I$ witness it is an $H$-set. Let $\sum c_{n} e^{i n x}=0$ off $E$. So by CantorLebesgue $c_{n} \rightarrow 0$. Fix a $C^{\infty}$ function $\varphi$ with $\hat{\varphi}(0)=1$ and $\operatorname{supp}(\varphi) \subseteq I$. Put $\varphi_{k}(x)$ $=\varphi\left(n_{k} x\right)$. Thus $\varphi_{k}=0$ on $E$. Let $S\left(\varphi_{k}\right) \cdot S \sim \sum C_{n}^{k} e^{i n x}$. By elementary calculations $C_{n}^{k} \rightarrow c_{n}$ as $k \rightarrow \infty$. By Lemma $5, \sum C_{n}^{k} e^{i n x}$ and $\varphi_{k}(x) \cdot \sum c_{n} e^{i n x}$ are equiconvergent. But $\varphi_{k}(x)=0$ on $E$ and $\sum c_{n} e^{i n x}=0$ off $E$; therefore $\sum C_{n}^{k} e^{i n x}=0, \forall x$, i.e. $C_{n}^{k}=0$. Therefore $c_{n}=0$.

We will finish this lecture by proving a key closure property of $\mathscr{U}$.

THEOREM 7 (Bary, 1923). The union of countably many closed $\mathscr{U}$-sets is in $\mathscr{U}$.

Corollary 8 (W. H. Young, 1909). If $C \subseteq \mathbb{T}$ contains no perfect set, then $C \in \mathscr{U}$. In particular, every countable set is in $\mathscr{U}$.

[Proof OF THE COROLlaRY. Else there is $S \sim \sum c_{n} e^{i n x}$ with $\sum c_{n} e^{i n x}=0$ off $C$ but $S \neq 0$. Let $B=\mathbb{T} \backslash\left\{x: \sum c_{n} e^{i n x}=0\right\} \subseteq C$. Then $B$ is Borel, so countable and in $\mathscr{M}$, contradicting Theorems 7 and 3.]

So we have the following picture (for Lebesgue measurable sets):

$$
\text { countable } \varsubsetneqq \mathscr{U} \varsubsetneqq \text { Lebesgue measure } 0
$$

(the second $\varsubsetneqq$ will be.proved later).

The proof of Bary's Theorem needs (a special case of) a result of de la ValleePoussin. We will see later that a trigonometric series $\sum c_{n} e^{i n x}$ may converge to 0 a.e. without being identically 0 . However we have

THEOREM 9 (de la Vallée-Poussin, 1912). Let $S \sim \sum c_{n} e^{i n x}$ have for each $x$ bounded partial sums

$$
S_{N}(x)=\sum_{-N}^{N} c_{n} e^{i n x}
$$

If $\sum c_{n} e^{i n x}=0$ a.e., then $S=0$.

The proof is rather technical and will be omitted.

ProOf of BARY'S TheOREM. Let $E_{n} \in \mathscr{U}, E_{n}$ closed. Put $E=\bigcup_{n} E_{n}$. Let $S \sim$ $\sum c_{n} e^{i n x}$ converge to 0 off $E$. Thus $\sum c_{n} e^{i n x}=0$ a.e. Clearly $c_{n} \rightarrow 0$. Assuming towards a contradiction that $S \neq 0$, let

$$
G=\left\{x: S_{n}(x) \text { is unbounded }\right\} .
$$

Then $G \subseteq E, G$ is $G_{\delta}$ and $G \neq \varnothing$ by Theorem 9. So $G$, in its relative topology, is Polish, and since $E_{i} \cap G=G_{i}$ is closed in $G$ and $G=\bigcup_{i} G_{i}$, by the Baire Category Theorem, there is an open interval $I_{0}$ and an index $i_{0}$ with $G \cap I_{0}=G_{i_{0}} \cap I_{0} \neq \varnothing$. It is enough to show that $\sum c_{n} e^{i n x}=0$ on $I_{0}$ (because then $I_{0} \cap G=\varnothing$ ). 
Fix $\varphi \in C^{\infty}$ with $\varphi>0$ on $I_{0}$ and $\varphi=0$ off $I_{0}$. Put $T=S(\varphi) \cdot S$. By Rajchman multiplication it is enough to show that $T$ converges to 0 everywhere, or, as $E_{i_{0}} \in \mathscr{U}$, just off $E_{i_{0}}$. Let $x \notin E_{i_{0}}$. It is enough again to assume $x \in I_{0} \cap E$, since $T$ certainly converges to 0 off $I_{0} \cap E$. So let $J \subseteq I_{0}$ be an interval with $x \in J$ and $\bar{J} \cap E_{i_{0}}=\varnothing$. Choose $\psi \in C^{\infty}$ with $\psi(x)=1$ and $\operatorname{supp}(\psi) \subseteq \bar{J}$. Now $T^{\prime}=S(\psi) \cdot T$ converges a.e. to 0 (as $S$, therefore $T$ does) and has bounded partial sums off $\bar{J} \cap G=\bar{J} \cap G_{i_{0}}=\varnothing$, i.e. everywhere. So, by Theorem $9, T^{\prime}=0$ and thus $T$ converges to 0 at $x$.

Recall here the

UNION Problem. Is the union of two or countably many Borel $\mathscr{U}$-sets a $\mathscr{U}$-set?

This is open even for two $G_{\delta}$ 's. Kholshchevnikova [25] showed that the answer is positive for two disjoint $G_{\delta}$ 's. One needs some definability restriction, as $\mathbb{T}$ can be written as the union of two (disjoint) sets containing no perfect subset.

Lecture VI. Elements of the modern theory. The Salem-Zygmund Theorem. The classification of $U$ and $U_{0}$. The modern theory of closed sets of uniqueness, on which we will be mainly concentrating from now on, is based on a reformulation of this concept in terms of functional analysis, originating in work of PiatetskiShapiro.

Recall that $A=A(\mathbb{T})$ is the Banach algebra of continuous functions on $\mathbb{T}$ with absolutely convergent Fourier series and norm $\|f\|_{A}=\sum|\hat{f}(n)|$. (Thus it is the same as $l^{1}=l^{1}(\mathbb{Z})$ with convolution.) The dual of $A$ (which is the same as $l^{\infty}$ ) is denoted by $P M$ - the space of pseudomeasures. Its norm is

$$
S=\left\{c_{n}\right\} \in P M \mapsto\|S\|_{\infty}=\sup _{n \in \mathbb{Z}}\left|c_{n}\right|
$$

The predual of $A$ (which is the same as $c_{0}$ ) is denoted by $P F$ - the space of pseudofunctions. Its norm is again $\|S\|_{\infty}$. (Note: $P F$ is a closed subspace of $P M$.) The duality of $P F, A$ and $A, P M$ is given by

$$
\langle f, S\rangle=\langle S, f\rangle:=\sum_{n \in \mathbb{Z}} \hat{f}(n) S(-n)
$$

(Note: $\hat{S}(n):=\left\langle e^{-i n x}, S\right\rangle=S(n)$ for $S \in P M$.)

EXAMPLES. If $f \in L^{1}=L^{1}(\mathbb{T})$ then $\hat{f} \in P F$ (Riemann-Lebesgue). Often we identify $f$ with $\hat{f}$ (as $f \mapsto \hat{f}$ is $1-1)$.

If $\mu \in M=M(\mathbb{T})(:=$ the space of (complex, Borel) measures on $\mathbb{T}=$ dual of $C(\mathbb{T}))$, then $\hat{\mu}(n)=\int e^{-i n x} d \mu(x)$ are the Fourier-Stieltjes coefficients of $\mu$. Then $|\hat{\mu}(n)| \leq\|\mu\|_{M}$, so $\hat{\mu} \in P M$ and $\|\hat{\mu}\|_{\infty} \leq\|\mu\|_{M}$. Again often we identify $\mu$ with $\hat{\mu}$ (as $\mu \mapsto \hat{\mu}$ is $1-1)$.

Given a trigonometric series $S \sim \sum c_{n} e^{i n x}$ with bounded coefficients, we can identify it with the pseudomeasure $S(n)=c_{n}$. So $P M \equiv$ the space of trigonometric series with bounded coefficients. With this identification Rajchman multiplication looks as follows: Given $S \in P M$ and $f \in A$, one defines $f \cdot S \in P M$ by

$$
\langle g, f \cdot S\rangle=\langle g f, S\rangle \text {. }
$$

It is easy to see that the trigonometric series of $f \cdot S$ is exactly $S(f) \cdot S$. Thus Lemma 4 of Lecture $\mathrm{V}$ reads: $S \in P F, f \in A \Rightarrow f \cdot S \in P F$. 
It remains to see what convergence of $S \sim \sum c_{n} e^{i n x}$ to 0 off a given set $E$ means in terms of the pseudomeasure $S$. This can be nicely done if $E$ is closed. We need to introduce the basic notion of support of a pseudomeasure.

Given $S \in P M$ and $V \subseteq \mathbb{T}$ open, we say that $S$ vanishes on $V$ if $\langle f, S\rangle=0$ for all $f \in A$ with $\operatorname{supp}(f) \subseteq V$. By partition of unity and compactness arguments, there is a largest open $V$ on which $S$ vanishes. Its complement is called the support of $S$, $\operatorname{supp}(S)$.

The following result relates convergence of $\sum c_{n} e^{i n x}$ to 0 and $\operatorname{supp}(S)$.

Theorem 1 (Piatetski-Shapiro [32], Kahane and Salem [8]). Let $S \in P F$, and let $E \subseteq \mathbb{T}$ be closed. Then the following are equivalent:

(i) $\sum S(n) e^{i n x}=0, \forall x \notin E$.

(ii) $\operatorname{supp}(S) \subseteq E$.

The proof uses the Riemann theory. From this we immediately obtain the following basic reformulation of closed sets of uniqueness (or, equivalently, multiplicity).

Theorem 2 (Piatetski-Shapiro [32], Kahane and Salem [8]). Let $E \subseteq \mathbb{T}$ be closed. Then $E$ is a set of multiplicity iff E supports a nonzero pseudofunction.

Denote $U:=\mathscr{U} \cap K(\mathbb{T})$ and $M:=\mathscr{M} \cap K(\mathbb{T})$, the classes of closed sets of uniqueness and multiplicity respectively. Thus

$$
E \in M \Leftrightarrow \exists S \in P F(S \neq 0 \wedge \operatorname{supp}(S) \subseteq E) .
$$

We introduce next the important class of sets of extended uniqueness.

DEFINITION. A Rajchman measure is a measure $\mu \in M(\mathbb{T})$ which is in $P F$ (i.e. $\hat{\mu} \in P F)$.

For example, $\lambda$ is Rajchman. Also the Rajchman measures form a band, i.e. $\mu \ll v$ and $v$ Rajchman $\Rightarrow \mu$ Rajchman (Milicer-Gružewska). So, as $|\mu| \ll \mu, \mu$ is Rajchman iff $|\mu|$ is Rajchman. So for most purposes one restricts attention to positive or even probability Rajchman measures.

Definition. A set $P \subseteq \mathbb{T}$ is called a set of extended uniqueness if for every probability Rajchman measure $\mu, \mu(P)=0$. Else it is called a set of restricted multiplicity. The corresponding classes of sets are denoted by $\mathscr{U}_{0}$ and $\mathscr{M}_{0}$.

It is easy to see that for universally measurable $P, P \in \mathscr{U} \Rightarrow P \in \mathscr{U}_{0}$. So for such sets we have

$$
\begin{aligned}
& \text { countable } \varsubsetneqq \mathscr{U} \varsubsetneqq \mathscr{U}_{0} \varsubsetneqq \text { Lebesgue measure } 0 \text {. } \\
& \text { (1) (2) }
\end{aligned}
$$

(1) (for actually closed sets) is due to Piatetski-Shapiro [32].

(2) (for actually closed sets) is due to Menshov (1916).

Put also $U_{0}:=\mathscr{U}_{0} \cap K(\mathbb{T})$ and $M_{0}:=\mathscr{M}_{0} \cap K(\mathbb{T})$. (Thus $K_{\omega}(\mathbb{T}) \varsubsetneqq U \varsubsetneqq U_{0} \varsubsetneqq I_{\lambda}$.)

The following are also equivalent formulations of the notion of (Borel) $\mathscr{U}_{0}$-set (or rather $\mathscr{M}_{0}$-set).

Proposition 3. Let $E \subseteq \mathbb{T}$ be Borel. Then the following are equivalent:

(i) $E \in \mathscr{M}_{0}$.

(ii) There is (probability) Rajchman measure $\mu$ with $\mu(E) \neq 0$.

(iii) E supports a probability Rajchman measure.

(iv) There is a (probability) measure $\mu \neq 0$ with $\sum \hat{\mu}(n) e^{i n x}=0$ off $E$. 
We come now to the famous Salem-Zygmund Theorem, which solves positively the characterization problem for perfect symmetric sets of constant ratio of dissection.

Definition. Given $0<\xi<1 / 2$, let $E_{\xi}$ denote the perfect set constructed like the Cantor set on $[0,2 \pi]$ except that the ratio $\xi$ is used instead of $1 / 3$ at each subdivision.

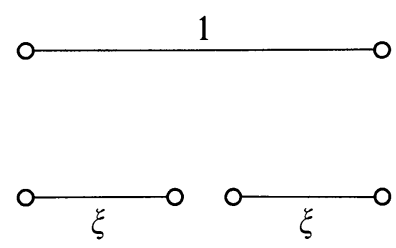

Definition. A real number $\theta$ is called a Pisot number if $\theta>1$ and $\theta$ is an algebraic integer all of whose conjugates have absolute value $<1$.

EXAMPLES. $2,3,4, \ldots ;(1+\sqrt{5}) / 2$.

Intuitively $\theta$ can be thought as a number whose powers $\theta^{n}$ approach integers (look at $\theta^{n}+\theta_{1}^{n}+\cdots+\theta_{p-1}^{n}$, where the $\theta_{i}$ are the conjugates of $\theta$ ). The remarkable fact about Pisot numbers is that they form a closed set! (Salem).

THEOREM 4 (Salem and Zygmund; see [37]). Let $0<\xi<1 / 2$. Then

$$
E_{\xi} \in U \Leftrightarrow E_{\xi} \in U_{0} \Leftrightarrow \theta=1 / \xi \text { is Pisot. }
$$

The proof proceeds by showing that if $\theta$ is Pisot, then $E_{\xi}$ is a particular type of $U$-set called an $H^{(n)}$-set $\left(H^{(1)}=H\right.$ ), a concept due to Piatetski-Shapiro. Conversely, if $\theta$ is not Pisot one shows that $E_{\xi} \notin U_{0}$, by showing that the standard measure on $E_{\xi}$ (coming from its identification with $2^{\omega}$ ) is Rajchman.

The following extension is also due to Salem and Zygmund.

Given $\left(\eta_{0}=0\right)<\eta_{1}<\eta_{2}<\cdots<\eta_{k}\left(<\eta_{k+1}=1\right)$, put $\xi=1-\eta_{k}$ and assume $\xi<\eta_{i+1}-\eta_{i}$ for $i<k$. The homogeneous perfect set associated with $\left(\xi ; \eta_{1}, \ldots, \eta_{k}\right)$ and denoted by $E\left(\xi ; \eta_{1}, \ldots, \eta_{k}\right)$ is defined by performing, starting from $[0,2 \pi]$, the following dissection:

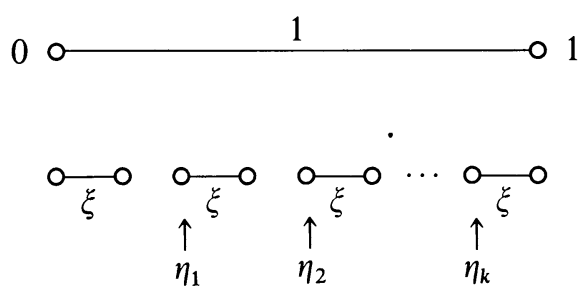

Then we have

THEOREM 5 (Salem and Zygmund; see [37]).

$$
\begin{aligned}
E\left(\xi ; \eta_{1}, \ldots, \eta_{k}\right) \in U & \Leftrightarrow E\left(\xi ; \eta_{1}, \ldots, \eta_{k}\right) \in U_{0} \\
& \Leftrightarrow \theta=1 / \xi \text { is Pisot } \wedge \eta_{1}, \ldots, \eta_{k} \in \mathbb{Q}(\theta) .
\end{aligned}
$$

We immediately have from Theorem 4 
COROLlaRy 6(Menshov, 1916). There is a closed set $E$ of Lebesgue measure 0 in $M_{0}$ (and hence also in $\left.M\right)$.

Proof. Take $E=E_{\xi}$ with $\theta=1 / \xi$ not Pisot.

We can use now the second Salem-Zygmund Theorem to calculate the complexity of $U$ and $U_{0}$.

THEOREM 7 (Solovay [33], Kaufman [13]). The sets $U$ and $U_{0}$ are complete $\Pi_{1}^{1}$ in $K(\mathbb{T})$ (so in particular non-Borel).

Proof. It is not hard to calculate, using Theorems 2 and 3, that $M$ and $M_{0}$ are $\Sigma_{1}^{1}$.

Since $U$ and $U_{0}$ are $\sigma$-ideals of closed sets, it is enough to show, by the dichotomy theorem, that $U$ and $U_{0}$ are not $\boldsymbol{\Pi}_{2}^{0}$. Clearly $\mathbb{Q} \cap[0,1]$ is not $\boldsymbol{\Pi}_{2}^{0}$. So it is enough to find continuous $f:[0,1] \rightarrow K(\mathbb{T})$ with

$$
x \in \mathbb{Q} \Leftrightarrow f(x) \in U \Leftrightarrow f(x) \in U_{0} .
$$

Put $f(x)=E(1 / 4 ; 3 / 8+x / 9,3 / 4)=E_{x}$,

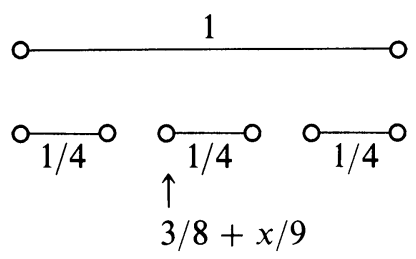

It is easy to show that $f$ is continuous. By Salem-Zygmund,

$$
E_{x} \in U \Leftrightarrow E_{x} \in U_{0} \Leftrightarrow x \in \mathbb{Q},
$$

since 4 is a Pisot number. (This argument actually uses a simpler case of Salem-Zygmund which is easier to prove.)

This theorem has obvious negative implications for the characterization problem. One cannot characterize when a closed (or perfect) set $E$ is in $U$ or $M$ (resp. $U_{0}$ or $M_{0}$ ) in terms of structural properties of $E$ which are "explicit" enough to be expressed in terms of countable operations given any reasonable description of $E$, for example its sequence of contiguous intervals.

Where is the dividing line between "explicit characterizability" and the lack of it?

(1) $E_{\xi}:$ "characterizable",

(2) $E_{\xi_{1}, \xi_{2}, \ldots}$ (symmetric perfect sets of variable dissection ratios): open,

(3) general $E$ : "uncharacterizable".

Concerning (2) the following is open: Is $\left\{E_{\xi_{1}, \xi_{2}, \ldots} \in U\right\}$ Borel in $K(\mathbb{T})$ ? We will see later, however, that $\left\{E_{\xi_{1}, \xi_{2}, \ldots} \in U_{0}\right\}$ is Borel.

Lecture VII. The structure of the $\sigma$-ideals $U$ and $U_{0}$ : Part 1. We will discuss here the basic definability and structural properties of the $\sigma$-ideals $U$ and $U_{0}$.

We start with the structural property we called calibration, an inner regularity property of $\sigma$-ideals. Recall that a $\sigma$-ideal $I$ of closed sets is calibrated iff for any closed set $E$ and sequence $E_{n} \in I$, if all closed subsets of $E \backslash \bigcup_{n} E_{n}$ are in $I$, so is $E$. 
As we have seen in the first four lectures, this property plays an important role in the structure theory of $\sigma$-ideals of closed sets.

Since $U_{0}$ is the class of null sets for a class of measures, it follows by the inner regularity of measures that $U_{0}$ is calibrated. We establish now the same fact for $U$.

THEOREM 1 (Kechris and Louveau [20], Debs and Saint Raymond [4]). The $\sigma$-ideal $U$ is calibrated.

Proof. We need first to express the Piatetski-Shapiro reformulation in dual terms. (This is actually the way Piatetski-Shapiro expressed it originally.)

THEOREM 2 (Piatetski-Shapiro [32]). Let $E \in K(\mathbb{\pi})$. Then

$$
E \in U \Leftrightarrow J(E) \text { is weak*-dense in } A,
$$

where $J(E)=\{f \in A$ : $f$ vanishes in an (open) neighborhood of $E\}$. (The weak*topology on $A$ is the one induced by its duality with $P F$.)

Proof. We are asserting that $E \in M \Leftrightarrow \overline{J(E)}^{w *} \neq A$, which by Hahn-Banach is equivalent to the existence of $S \in P F, S \neq 0$, with $\langle f, S\rangle=0$ for all $f \in J(E)$, i.e. $\operatorname{supp}(S) \subseteq E$.

The proof of Theorem 1 is a simple application of the "shrinking method", a technique of using multiplication of a pseudomeasure $S$ by a function $f$ in $A$ to shrink appropriately the support of $S$ "without losing much of $S$ ". Keep in mind always the simple fact that

$$
\operatorname{supp}(f \cdot S) \subseteq \operatorname{supp}(f) \cap \operatorname{supp}(S) .
$$

The basic lemma for our application here is

Lemma 3. Let $S \in P F, E \in U$ and $\varepsilon>0$. There is $f \in J(E)$ such that $\|S-f \cdot S\|_{\infty}$ $<\varepsilon$ and $\operatorname{supp}(f \cdot S) \cap E=\varnothing$.

Proof. By Hahn-Banach if $Z \subseteq A$ is convex, then for $S \in P F, \bar{Z}^{w^{*}} \cdot S \subseteq \overline{Z \cdot S}$. So if $Z=J(E), 1 \in \widetilde{J(E)}^{w^{*}}$ by Theorem 2 , so $1 \cdot S=S \in \widehat{J(E) \cdot S}$.

We complete now the proof of Theorem 1 as follows: Say $E_{n} \in U$ and every closed subset of $E \backslash \bigcup_{n} E_{n}$ is in $U$, where $E \in K(\mathbb{T})$, but $E \notin U$, towards a contradiction. Let $S \in P F,\|S\|_{\infty}=1$ and $\operatorname{supp}(S) \subseteq E$. By Lemma 3 find $S_{n} \in P F$ with $S_{0}=S$, $\left\|S_{n}-S_{n+1}\right\|_{\infty}<2^{-n-2}$ and $\operatorname{supp}\left(S_{n+1}\right) \subseteq \operatorname{supp}\left(S_{n}\right) \backslash E_{n}$. Then $\lim S_{n}=T \in P F$ and $T \neq 0$. Also $\operatorname{supp}(T) \subseteq \bigcap_{n} \operatorname{supp}\left(S_{n}\right) \subseteq E \backslash \bigcap_{n} E_{n}$, a contradiction.

One corollary of the calibration property is that the union of countably many $\Sigma_{3}^{0}=G_{\delta \sigma}$ sets of interior uniqueness is also of interior uniqueness. (A set of interior uniqueness is one which contains no closed $M$-sets). Recall now the

INTERIOR PROBLEM. Is every $\Sigma_{3}^{0}$ (equivalently, an arbitrary) set of interior uniqueness a set of uniqueness?

Thus we see that a positive answer to the interior problem implies a positive answer to the union problem for $\Sigma_{3}^{0}$ sets.

We now discuss definability properties of the $\sigma$-ideals $U$ and $U_{0}$.

First, as $U$ and $U_{0}$ are $\Pi_{1}^{1}$ sets, they admit $\Pi_{1}^{1}$-ranks. We look for canonical ones.

Piatetski-Shapiro has defined a canonical rank for $U$-sets as follows:

Recall that for $E \in U$, the ideal $J(E)$ is weak*-dense in $A$. Since the weak*topology is not metrizable, weak*-closures cannot in general be obtained by just taking weak*-limits of sequences. But by results of Banach, transfinite iteration of 
this process suffices. Define therefore inductively for each $E \in U$ :

$$
\begin{aligned}
J^{0}(E) & =J(E), \\
J^{\alpha+1}(E) & =\text { the set of weak } * \text {-limits of sequences from } J^{\alpha}(E), \\
J^{\lambda}(E) & =\bigcup_{\alpha<\lambda} J^{\alpha}(E), \lambda \text { limit. }
\end{aligned}
$$

Then by a theorem of Banach this process terminates at a countable ordinal $\alpha_{0}$, and $J^{\alpha_{0}}(E)=\overline{J(E)}^{w^{*}}$. So if $E \in U$ there is countable $\alpha$ with $J^{\alpha}(E)=A$. We call the least such $\alpha$ the Piatetski-Shapiro rank of $E,[E]_{\mathbf{P S}}$.

THEOREM 4 (Solovay [34]). The Piatetski-Shapiro rank is a $\Pi_{1}^{1}$-rank on $U$.

The original proof by Solovay used effective descriptive set theory and boundedness arguments in admissible sets. Kechris and Louveau found two other ranks on $U$, which they have shown to be the same as $[E]_{\mathrm{PS}}$, one "living" in $P F$ and the other in $P M\left([E]_{\mathrm{PS}}\right.$ "lives" in $\left.A\right)$. The " $P F$-rank" provides a straightforward proof that $[E]_{\mathrm{PS}}$ is a $\Pi_{1}^{1}$-rank, but the proof of its equivalence with $[E]_{\mathrm{PS}}$ requires a fair amount of technical analytical work.

Thus we have a canonical hierarchy of $U$-sets with at most $\omega_{1}$ levels. In fact we have exactly $\omega_{1}$ levels, as follows from the fact that $U$ is not Borel and the easy half of the following boundedness theorem.

TheOREM 5. If $X$ is Polish, $P \subseteq X$ is $\Pi_{1}^{1}$ and $\varphi: P \rightarrow \omega$ is a $\Pi_{1}^{1}$-rank, then

$$
P \text { is Borel } \Leftrightarrow \exists \alpha<\omega_{1} \forall x \in P(\varphi(x) \leq \alpha) \text {. }
$$

(The unboundedness of the Piatetski-Shapiro rank was originally proved by a direct construction by McGehee [31].)

The "simplest" sets in the hierarchy of $U$-sets are those of rank 1, i.e. those for which $J(E)$ is sequentially weak *-dense in $A$, i.e. there is $f_{n} \in J(E)$ with $f_{n} \rightarrow^{w^{*}} 1$. The countable closed sets have PS-rank 1 , as do all $H^{(n)}$-sets (e.g. $\left.E_{1 / 3}\right)$ as well as most explicitly constructed $U$-sets. Sets of PS-rank 1 are denoted by $U^{\prime}$. By a result of Banach they can be characterized in a more "quantitative" way as follows: For $S \in P M$, let

$$
R(S)=\varlimsup \lim |S(n)|
$$

Thus $S \in P F \Leftrightarrow R(S)=0$, and it is easy to see that $R(S)=$ distance of $S$ from $P F$. For $E \in K(\mathbb{T})$, let

$$
\eta(E)=\inf \left\{R(S):\|S\|_{\infty}=1, S \in P M, \operatorname{supp}(S) \subseteq E\right\} .
$$

Then $E \in U^{\prime} \Leftrightarrow \eta(E)>0$.

Piatetski-Shapiro used $[E]_{\mathrm{PS}}$ to prove a decomposition theorem of the form: Every $E \in U$ can be written as $E=\bigcup_{n} E_{n}$, where $E_{n}$ are $U$-sets of some "simpler" type. In fact these $E_{n}$ are almost - but not quite!- $U^{\prime}$-sets. Can they actually be $U^{\prime}-$ sets? Every $U$-set known until recently had this property, but we will see later that the answer is in general negative.

We turn now to the question of a canonical $\Pi_{1}^{1}$-rank on $U_{0}$. Such a rank was first found by Kechris and Louveau, motivated by one of their reformulations of the PS-rank on $U$. This rank on $U_{0}$ will be denoted by $[E]_{0}$. An equivalent description of $[E]_{0}$ found later by Debs and Saint Raymond resembles $[E]_{\mathrm{PS}}$ : Given $E \in K(\mathbb{T})$, 
let $I_{\text {neg }}(E)=\{f \in A: \operatorname{Re}(f) \leq 0$ on $E\}$. Then $E \in U_{0} \Leftrightarrow \overline{I_{\text {neg }}(E)^{w^{*}}}=A \Leftrightarrow 1 \in \overline{I_{\text {neg }}(E)^{w^{*}}}$. Put $[E]_{0}^{*}=$ least $\alpha$ such that $1 \in I_{\text {neg }}^{\alpha}(E)$. Then $[E]_{0}=[E]_{0}^{*}$.

The sets of rank 1 here are denoted by $U_{0}^{\prime}$. They can be also characterized as follows: For $E \in K(\mathbb{T})$, let $\eta_{0}(E)=\inf \{R(\mu): \mu$ a probability measure supported by $E\}$. Then $E \in U_{0}^{\prime} \Leftrightarrow \eta_{0}(E)>0$.

As opposed to $U^{\prime}$ and $U$, it turned out that $U_{0}^{\prime}$ is a (Borel of course) basis for $U_{0}$.

THEOREM 6 (Kechris and Louveau [20]). Every $U_{0}$-set is a countable union of $U_{0}^{\prime}$-sets.

Thus in particular one has, according to results in the first four lectures, a Cantor-Bendixson type rank $\mathrm{rk}_{U_{0}^{\prime}}$ on $U_{0}$ corresponding to the basis $U_{0}^{\prime}$. This turns out to be equal to $[E]_{0}$ again.

The idea of the proof of Theorem 6 is the following: By simple manipulations it is enough to show that if $E \in K(\mathbb{T})$ has the property that every nonempty portion $V \cap E$ ( $V$ open) of $E$ supports probability measures with arbitrarily small $R(\mu)$, then $E \in M_{0}$. Given such an $E$, one shows by standard weak*-approximation arguments that for each $\varepsilon>0$ the probability measures supported by $E$ with $R(\mu)<\varepsilon$ are weak*-dense in the probability measures supported by $E$. Then one constructs inductively, by appropriate "iterating and averaging" procedures, a sequence $\mu_{1}$, $\mu_{2}, \ldots$ of probability measures on $E$ and a sequence $0<n_{1}<n_{2}<\cdots$ such that

$$
\sup \left\{\left|\hat{\mu}_{k}(m)\right|:|m| \geq n_{i}\right\}<2^{-i}, \quad \forall k \geq i .
$$

Then if $\mu$ is a weak*-limit of a subsequence of the $\mu_{k}$ 's, $\hat{\mu}$ is in $P F$ and a probability measure supported by $E$.

In conclusion, we have seen that both $U$ and $U_{0}$ have canonical $\Pi_{1}^{1}$-ranks. This gives for each one of them a canonical hierarchy consisting of $\omega_{1}$ distinct levels. The sets $U^{\prime}$ and $U_{0}^{\prime}$ of rank 1 receive particular attention because of their "simplicity" and because most explicit examples belong there. The class $U_{0}^{\prime}$ forms a Borel basis for $U_{0}$, but as we will see in the next lecture the class $U^{\prime}$ does not form a Borel basis for $U$.

One can also use these $\Pi_{1}^{1}$-ranks to show that $U$ and $U_{0}$ are even "locally" non-Borel.

THEOREM 7 (Debs and Saint Raymond [4], Kaufman [14], [15], Kechris and Louveau [20]). Let I be $U$ or $U_{0}$. Then for each $E \in K(\mathbb{T}) \backslash I$, the $\sigma$-ideal $K(E) \cap I$ is $\Pi_{1}^{1}$-complete.

This can be proved for example by showing that the canonical $\Pi_{1}^{1}$-rank on $I$ is unbounded in $K(E) \cap I$. The construction is based on a key "shrinking argument" due to Kaufman.

Lecture VIII. The structure of the $\sigma$-ideals $U$ and $U_{0}$. Part 2: Applications. The Borel basis problem for $U$ and $U_{0}$ was raised in the paper of Kechris, Louveau and Woodin discussed in the first four lectures. Although $U$ and $U_{0}$ are not Borel and therefore not "simply characterizable", there are other examples of classes of thin sets (e.g. the countable closed ones) which although they form a non-Borel class, can still be decomposed into "simply characterizable" (i.e. Borel) components (e.g. singletons). 
We have seen in the preceding lecture that the Borel basis problem admits a positive solution for $U_{0}$. Soon after this was established, Debs and Saint Raymond solved this problem negatively for $U$.

THEOREM 1 (Debs and Saint Raymond [4]). The $\sigma$-ideal $U$ of closed uniqueness sets has no Borel basis.

We will sketch the ideas of the proof. The key result from analysis that is used is a deep result of Körner [26] on the existence of so-called Helson sets of multiplicity. For our purposes here this result can be considered as a very strong form of Piatetski-Shapiro's result that $U \varsubsetneqq U_{0}$. We will state Körner's Theorem in a weaker version which is sufficient for Theorem 1.

Definition. Define the class $U_{1}^{\prime}$ of closed sets as follows: For $E \in K(\mathbb{T})$ let $I(E)$ be the class of functions in $A$ which vanish on $E$ (not in a neighborhood of $E$ ). Then let

$$
E \in U_{1}^{\prime} \Leftrightarrow \exists\left\{f_{n}\right\}\left(f_{n} \in I(E) \& f_{n} \rightarrow^{w^{*}} 1\right) .
$$

One can see that $U^{\prime} \subseteq U_{1}^{\prime} \subseteq U_{0}^{\prime}$. (These sets first came up in the PiatetskiShapiro decomposition theorem mentioned earlier: If $E \in U$, then $E=\bigcup_{n} E_{n}$, where $E_{n} \in U \cap U_{1}^{\prime}$.)

Now one has

THEOREM 2 (Körner [26]). $U_{1}^{\prime} \nsubseteq U$.

(It turns out also that $U_{0}^{\prime} \nsubseteq\left(U_{1}^{\prime}\right)_{\sigma}$ (Piatetski-Shapiro).)

Thus there are $M$-sets which are in $U_{1}^{\prime}$, and thus they are "almost" $U^{\prime}$-sets. The proof of Körner's Theorem was originally very complicated. Kaufman [12] found another proof (using some of the ideas in Piatetski-Shapiro's proof that $U \varsubsetneqq U_{0}$ ), which is much simpler although still very subtle. It is based on a "shrinking argument" and works within any given $M$-set.

Fix now $E \in M, E \in U_{1}^{\prime}$. By a simple argument we can actually assume that $\overline{E \cap V} \in M$ for every nonempty portion $E \cap V$ of $E$ ( $V$ open). Recall now the basis theorem from the first four lectures:

If $I$ is a $\sigma$-ideal in $K(E)$ with basis $B$ and $I$ is calibrated, while for each nonempty portion $E^{\prime}=E \cap V$ of $E$ we have $B \cap K\left(E^{\prime}\right) \neq I \cap K\left(E^{\prime}\right)$, then every dense $G_{\delta}$ of $E$ is not in $I_{\mathrm{int}}$.

Applying this to $I=K(E) \cap U$, which we already know is calibrated, we see that if it had a Borel basis $B$, then $B \cap K\left(E^{\prime}\right) \neq I \cap K\left(E^{\prime}\right)$, as $I \cap K\left(E^{\prime}\right)$ is not Borel (by Theorem 7 of Lecture VII) while $B \cap K\left(E^{\prime}\right)$ is, so every dense $G_{\delta}$ in $E$ is not in $U_{\text {int }}$.

So it is enough to show that there is a dense $G_{\delta}$ set $G$ in $E$ which is in $U_{\text {int }}$. This can be done as $E$ is "almost" a $U$-set. To construct $G$, fix a dense sequence $\left\{x_{p}\right\}$ in $E$. Fix also, as $E \in U_{1}^{\prime}$, a sequence $f_{n} \in I(E)$ with $f_{n} \rightarrow^{w^{*}} 1$. It is a general fact about $A$ that if $f \in A, a \in \mathbb{T}$ and $f(a)=0$, then for $\varepsilon>0$ there is $g \in A$ with $g=0$ in a neighborhood of $a$ and $\|f-f g\|_{A}<\varepsilon$. Using this, for each fixed $n$, define inductively on $p$ a function $f_{n, p} \in I(E)$ and an open neighborhood $V_{n, p}$ of $x_{p}$ with

$$
\begin{gathered}
f_{n, p}=0 \quad \text { on } \bigcup_{q \leq p} V_{n, q}, \\
\left\|f_{n, p}-f_{n}\right\|_{A} \leq 2^{-n}, \quad\left\|f_{n, p}-f_{n, p-1}\right\|_{A} \leq 2^{-p} .
\end{gathered}
$$


Put $V_{n}=\bigcup_{p} V_{n, p}$ and $h_{n}=\lim _{p} f_{n, p}$. Then $h_{n}=0$ on $V_{n} \supseteq\left\{x_{n}: n \in \mathbb{N}\right\}$ and $\left\|h_{n}-f_{n}\right\|_{A}$ $\leq 2^{-n}$; thus $h_{n} \rightarrow^{w^{*}} 1$ as well. Put $G=\bigcap_{n}\left(E \cap V_{n}\right)$. Then $G$ works, as for each closed $F \subseteq G$ the sequence $h_{n}$ vanishes on $V_{n} \supseteq F$ and $h_{n} \rightarrow^{w^{*}} 1$, so actually $F \in U^{\prime}$.

We can summarize now the basic structural and definability properties of the $\sigma$-ideals $U$ and $U_{0}$ :

(A) They are calibrated $\sigma$-ideals,

(B) They are $\Pi_{1}^{1}$ but locally non-Borel,

(C)

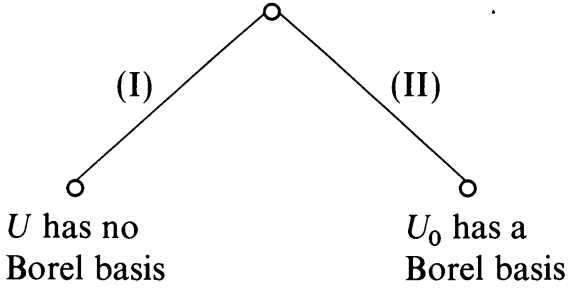

(This is the first structural difference between $U$ and $U_{0}$.)

We discuss now various applications of this theory.

(I) (1) The fact that $U$ has no Borel basis has clear negative implications for the characterization problem: One cannot even decompose $U$-sets into "simply characterizable" components.

(2) On the other hand, the non-basis result can be used to prove existence theorems. For example, the $U^{\prime}$-sets form a Borel class, so there are $U$-sets which are not countable unions of $U^{\prime}$-sets. This gives the first new examples of $U$-sets in a long time, since every $U$-set known until recently was known to be a countable union of $U^{\prime}$-sets. In particular, as $U^{\prime} \supseteq \bigcup_{n} H^{(n)}$ one has $U$-sets which are not unions of $H^{(n)}$-sets even with varying $n$. (For fixed $n$ this is a difficult theorem of Piatetski-Shapiro [32], with a totally different proof. The general result was conjectured by Piatetski-Shapiro in [32].)

(II) (1) Since $U_{0}^{\prime}$ is a basis for $U_{0}$, it follows easily that every perfect symmetric set $E_{\xi_{1}, \xi_{2}, \ldots}$ which is in $U_{0}$ is actually in $U_{0}^{\prime}$. Then

$$
\left\{E_{\xi_{1}, \xi_{2}, \ldots} \in U_{0}\right\}=\left\{E_{\xi_{1}, \xi_{2}, \ldots} \in U_{0}^{\prime}\right\}
$$

is a Borel class. This perhaps suggests that there could be a characterization of the $E_{\xi_{1}, \xi_{2}, \ldots}$ in $U_{0}$. This is however an open problem. (We repeat here that it is not known if $\left\{E_{\xi_{1}, \xi_{1}, \ldots} \in U\right\}$ is Borel or not.)

(2) The $\sigma$-ideal $U_{0}$ satisfies now all the hypotheses of the basis theorem and the theorem of Debs and Saint Raymond following that (these were discussed in the fourth lecture), so we have the following solution of the category problem.

THEOREM 3 (Debs and Saint Raymond [4]). Every $\mathscr{U}_{0}$-set $P \subseteq \mathbb{T}$ with the Baire property is of the first category. In fact if $P \subseteq \mathbb{T}$ is $\Sigma_{1}^{1}$ and in $\mathscr{U}_{0}$, there is a sequence $F_{n} \in U_{0}$ with $P \subseteq \bigcup_{n} F_{n}$.

For $P \in \Pi_{1}^{1}, P \in \mathscr{U}_{0}$ we know that $P$ is of the first category, but one cannot prove in $\mathrm{ZFC}$ that $P \subseteq \bigcup_{n} F_{n}$ with $F_{n} \in U_{0}$ ! This can be proved, however, assuming $\forall x \in \mathbb{R}\left(\aleph_{1}^{L[x]}<\aleph_{1}\right)$. It is not known if this covering property is equiconsistent with ZFC or requires large cardinal hypotheses.

(3) One can see now several old and new results of the theory of uniqueness for 
trigonometric series as immediate consequences of the fact that Borel $\mathscr{U}_{0}$-sets are of the first category. Here is a sampler:

(i) Menshov's Theorem. There are $M_{0}$-sets of Lebesgue measure 0 . To see this, recall the standard fact that there is a dense $G_{\delta}$ set $G \subseteq \mathbb{T}$ of measure 0 . Then $G \in$ $\mathscr{M}_{0}$, so by regularity $G$ contains an $M_{0}$-set. So Menshov's Theorem can be seen as a consequence of the "orthogonality" of measure and category!

(ii) Ivashev-Musatov's [7] and Kaufman's [11] Theorem. There are $M_{0}$-sets of $h$-Hausdorff measure 0 (for any $h$ ), within any $M_{0}$-set. Same proof.

(iii) Lyons' solution [28] of the Kahane-Salem problem [9]. Let $P$ be the set of nonnormal, say in base 10 , numbers. Thus, by Borel's theorem, $P$ has Lebesgue measure 0 . However, $P \in M_{0}$. For the proof, it is easy to check that $P$ is comeager.

We conclude with a summary of further developments concerning descriptive set theory and harmonic analysis:

(1) Kechris and Louveau [20] (see also [21]) found a simple analytical proof of the Debs and Saint Raymond covering theorem for $\Sigma_{1}^{1} U_{0}$-sets (and therefore its corollaries). The method is applicable in related contexts and leads to the following:

(A) It connects the union problem for $G_{\delta}$-sets to harmonic synthesis problems, and in particular it shows surprisingly that a counterexample (which is the most likely possibility) would have to use Körner's Theorem!

(B) It allows characterization of closed sets within which a "metric" condition in the form of Hausdorff $h$-measure 0 implies uniqueness, answering a question of Kaufmann (Dougherty and Kechris [5]).

(2) Methods of descriptive set theory have been applied to Lyons' work on (A) (Lyons [27]) the characterization of Rajchman measures by their null sets (i.e. $\mu$ is a Rajchman measure iff it annihilates every $U_{0}$-set) (Louveau and Mokobodzki; see [20]) and (B) (Lyons [29]) the failure of the so-called Rajchman conjecture (i.e. $\mu$ is a Rajchman measure iff it annihilates every $H$-set) (Kechris and Lyons [24], Kaufman [17]).

(3) One can analyze the gap between $U$ and $U_{0}$ by providing a transfinite hierarchy of classes filling this gap and relating it to definability problems concerning harmonic synthesis (Lyons [30], Kechris, Louveau and Tardivel [22]).

(4) One can establish that $U_{0}$ is "hereditarily" $\boldsymbol{\Pi}_{1}^{1}$-complete (Kechris [19]).

(5) Descriptive set theoretic studies of other types of exceptional sets or application of descriptive set theoretic methods to harmonic analysis are also the subject of Kaufman [16]-[18], Tardivel [35], [36], S. Kahane [10] and Becker, Kahane and Louveau [3]. (The last paper classifies certain well-known classes of thin sets as being actually $\Sigma_{2}^{1}$-complete!)

\section{REFERENCES}

[1] N. K. BARY, Sur l'unicité du développement trigonométrique, Fundamenta Mathematicae, vol. 9 (1927), pp. 62-115.

[2] - A treatise on trigonometric series. Vols. I, II, Macmillan, New York, 1964.

[3] H. BeCKer, S. KaHANe and A. Louveau, Some $\Sigma_{2}^{1}$-complete sets in harmonic analysis, Transactions of the American Mathematical Society (to appear).

[4] G. Debs and J. SaInt Raymond, Ensembles d'unicité et d'unicité au sens large, Annales de l'Institut Fourier (Grenoble), vol. 37 (1987), fasc. 3, pp. 217-239. 
[5] R. DOUGHERTY and A. S. KeCHRIS, Hausdorff measures and sets of uniqueness for trigonometric series, Proceedings of the American Mathematical Society, vol. 105 (1989), pp. 894-897.

[6] W. Hurewicz, Relative perfekte Teile von Punktmengen und Mengen (A), Fundamenta Mathematicae, vol. 12 (1928), pp. 78-109.

[7] O. S. IvaShev-MuSatov, M-sets and Hausdorff measure, Soviet Mathematics Doklady, vol. 3 (1962), pp. 213-216.

[8] J.-P. KAHANE and R. SALEM, Ensembles parfaits et séries trigonométriques, Hermann, Paris, 1963.

[9] - Distribution modulo 1 and sets of uniqueness, Bulletin of the American Mathematical Society, vol. 70 (1964), pp. 259-261.

[10] S. KaHANE, Ensembles de convergence absolue, ensembles de Dirichlet faibles et $\uparrow$-idéaux, Comptes

Rendus de l'Académie des Sciences, Séries I: Mathématique, vol. 310 (1990), pp. 355-357.

[11] R. Kaufman, Kronecker sets and metric properties of $M_{0}$-sets, Proceedings of the American Mathematical Society, vol. 36 (1972), pp. 519-524.

[12] - M-sets and distributions, Pseudofunctions and Helson sets, Astérisque, vol. 5, Société Mathématique de France, Paris, 1973, pp. 225-230.

[13] - Fourier transforms and descriptive set theory, Mathematika, vol. 31 (1984), pp. 336-339.

[14] - Absolutely convergent Fourier series and some classes of sets, Bulletin des Sciences Mathématiques, ser. 2, vol. 109 (1985), pp. 363-372.

[15] - Perfect sets and sets of multiplicity, Hokkaido Mathematical Journal, vol. 16 (1987), pp. $51-55$.

[16] - Continuous measures and analytic sets, Colloquium Mathematicum, vol. 58 (1989), pp. $17-21$.

[17] - Topics on analytic sets (preprint).

[18] - Bernoulli convolutions - an application of set theory in analysis, Colloquium Mathematicum, vol. 60/61 (1990), pp. 93-98.

[19] A. S. KECHRIS, Hereditary properties of the class of closed uniqueness sets, Israel Journal of Mathematics (to appear).

[20] A. S. KECHRIS and A. LOUVEAU, Descriptive set theory and the structure of sets of uniqueness, London Mathematical Society Lecture Note Series, vol. 128, Cambridge University Press, Cambridge, 1989.

[21] - Covering theorems for uniqueness and extended uniqueness sets, Colloquium Mathematicum, vol. 59 (1990), pp. 63-79.

[22] A. S. Kechris, A. LouveAu and V. TARDIVEL, The class of synthesizable pseudomeasures, Illinois Journal of Mathematics, vol. 35 (1991), pp. 107-146.

[23] A. S. Kechris, A. LouveAu and W. H. WoOdin, The structure of $\sigma$-ideals of compact sets, Transactions of the American Mathematical Society, vol. 301 (1987), pp. 263-288.

[24] A. S. KECHRIS and R. Lyons, Ordinal rankings on measures annihilating thin sets, Transactions of the American Mathematical Society, vol. 310 (1988), pp. 747-758.

[25] N. N. KholshCheVnikova, The sum of less-than-continuum many closed U-sets, Moscow University Mathematics Bulletin, vol. 36 (1981), no. 1, pp. 60-64.

[26] T. Körner, A pseudofunction on a Helson set. I, II, Pseudofunctions and Helson sets, Astérisque, vol. 5, Société Mathématique de France, Paris, 1973, pp. 3-224, 231-239.

[27] R. LyONS, Fourier-Stieltjes coefficients and asymptotic distribution modulo 1, Annals of Mathematics, ser. 2, vol. 122 (1985), pp. 155-170.

[28] - The measure of non-normal sets, Inventiones Mathematicae, vol. 83 (1986), pp. 605-616.

[29] - The sizes of some classes of thin sets, Studia Mathematica, vol. 86 (1987), pp. 59-78.

[30] - A new type of sets of uniqueness, Duke Mathematical Journal, vol. 57 (1988), pp. 431-458.

[31] O. C. MCGeHEE, A proof of a statement of Banach on the weak*-topology, Michigan Mathematical Journal, vol. 15 (1968), pp. 135-140.

[32] I. I. PiATETSKi-Shapiro, On the problem of uniqueness of expansion of a function in trigonometric series, Moskovskii Gosudarstvennyi Universitet Uchenye Zapiski, vol. 155 (Matematika, 5) (1952), pp. 54-72; Supplement, ibid., vol. 165 (Matematika, 7) (1954), pp. 79-97. (Russian)

[33] R. Solovay, Private communication, December 1983.

[34] - Private communication, February 1984.

[35] V. TARdivel, Ensembles de Riesz, Transactions of the American Mathematical Society, vol. 305 (1988), pp. 167-174. 
[36] - Fermés d'unicité dans les groupes abéliens localements compacts (to appear).

[37] A. Zygmund, Trigonometric series, 2nd rev. ed., Vols. 1, 2, reprint, Cambridge University Press, Cambridge, 1979.

\title{
DEPARTMENT OF MATHEMATICS \\ CALIFORNIA INSTITUTE OF TECHNOLOGY \\ PASADENA, CALIFORNIA 91125
}

E-mail:kechris@romeo.caltech.edu,kechris@caltech.bitnet

\author{
EQUIPE D'ANALYSE \\ UNIVERSITÉ PARIS-VI \\ 75230 PARIS, FRANCE
}

E-mail: louveau@frunip62.bitnet 